\title{
El uso de mascarilla en el deporte: una revisión integradora durante la Covid-19
}

\author{
The use of masks in sport: an integrative review during Covid-19 \\ Joel Manuel Prieto Andreu
}

Universidad Internacional de la Rioja

CORRESPONDENCIA:

Joel Manuel Prieto Andreu

joelmanuel.prieto@unir.net

Recepción: septiembre 2020 • Aceptación: enero 2021
CÓMO CITAR EL ARTÍCULO:

Prieto, J.M. (2021). El uso de mascarilla en el deporte: una revisión integradora durante la Covid-19. Cultura, Ciencia y Deporte, 16(49), 393-410. http://dx.doi.org/10.12800/ccd.v16i49.1554

\section{Resumen}

El uso de la mascarilla en el ámbito deportivo se ha estudiado para mejorar el rendimiento del deportista y como protección ante un ambiente contaminado, como el existente en el panorama pandémico actual de la Covid-19. La presente revisión analiza la repercusión de la mascarilla en diferentes factores fisiológicos mientras se hace ejercicio físico. Se hizo una selección aleatoria de 2420 estudios publicados en Google Académico, 118 en SCOPUS y de 137 publicados en WOS entre los períodos de 2016-2020, con el 95\% de confianza y +/4,3 de error muestral, el cual sirvió para la selección y análisis final de 10 estudios escogidos intencionalmente siguiendo los niveles de evidencia del CTFPHC y el rigor metodológico de un sistema de clasificación estructurado Ilamado PEDro Scale. Los resultados destacan que el uso de mascarillas en deportistas provoca respiración hipóxica e hipercápnica a una intensidad determinada; el uso de mascarillas como la Elevation Training Mask (ETM) entrena los músculos respiratorios, pero no mejora el rendimiento anaeróbico láctico, como sí lo hace el entrenamiento hipóxico intermitente (IHT) en altura. Se discute sobre las condiciones de hipoxia e hipercapnia y el uso de las mascarillas para la mejora del rendimiento físico en deportistas.

Palabras clave: mascarilla, deporte, hipoxia, hipercapnia, Covid-19.

\begin{abstract}
The use of the mask in the sports field has been studied to improve the athlete's performance and its protection against a contaminated environment, such as the one existing in the current pandemic panorama of Covid-19. This review analyzes the impact of the mask on different physiological factors while doing physical exercise. A random selection was made of 2,420 studies published in Scholar Google, 118 in SCOPUS and 137 studies published in WOS between the periods 2016-2020, with $95 \%$ confidence and +/- 4.3 sampling error, which served for the selection and final analysis of 10 studies chosen intentionally following CTFPHC levels of evidence and the methodological rigor of a structured classification system called PEDro Scale. The results highlight that the use of masks in athletes causes hypoxic and hypercapnic breathing at a certain intensity; the use of masks such as the Elevation Training Mask (ETM) trains the respiratory muscles but does not improve lactic anaerobic performance as intermittent hypoxic training (IHT) does in height. It is discussed about hypoxia and hypercapnia conditions and the use of masks to improve physical performance in athletes.
\end{abstract}

Key words: mask, sport, hypoxia, hipercapnia, Covid-19. 


\section{Introducción}

La investigación sobre el uso de mascarillas en el ámbito deportivo se puede concretar en dos vertientes: estudios que emplean las mascarillas para simular una altitud determinada para mejorar el rendimiento del deportista (Bellovary et al., 2019; Porcari et al., 2016; Sellers, Monaghan, Schnaiter, Jacobson, \& Pope, 2016) y estudios recientes que los emplean como factor de protección ante un ambiente contaminado o que examinan sus repercusiones fisiológicas en un grupo único o comparando un grupo con un determinado tipo de mascarilla con un grupo control (Freemas, Wilhite, Greenshields, Adamic, \& Mickleborough, 2020; Guo \& Fu, 2019; Jung, Lee, John, \& Lee, 2019; Pifarré, Zabala, Grazioli, \& Maura, 2020; Tobin, Costalat, \& Renshaw, 2020; Wagner \& Clark, 2016; Wong, 2020). En el panorama pandémico del año 2020, en el que se ha establecido la obligación de llevar mascarilla, esta revisión tiene el objetivo de analizar la literatura sobre el uso de la mascarilla, planteando cuáles podrían ser sus repercusiones mientras se hace ejercicio físico.

Hay estudios que analizan los efectos en los atletas al usar mascarillas especializadas que restringen el flujo de aire y simulan el entrenamiento en altitud. El entrenamiento en altitud y el entrenamiento de los músculos respiratorios (RMT) pueden mejorar el rendimiento en atletas de élite, se han desarrollado varios dispositivos para ayudar a los atletas a ganar ventaja en competición, como, por ejemplo, la Elevation Training Mask 2.0 (ETM). La ETM simula supuestamente el entrenamiento en altitud y se ha sugerido para aumentar la capacidad aeróbica $\left(\mathrm{VO}_{2} \mathrm{max}\right)$, el rendimiento de resistencia y la función pulmonar. Según los resultados de Porcari, et al. (2016) el uso del ETM puede mejorar los marcadores específicos del rendimiento de resistencia, el Volumen Máx. de Oxígeno $\left(\mathrm{VO}_{2} \max \right)$, el Umbral Ventilatorio (UV) y el Umbral de Compensación Respiratoria (UCR), más allá de las mejoras observadas solo con el entrenamiento por intervalos. Sin embargo, siguiendo a Jung, Lee, John, y Lee (2019) se necesitan estudios futuros para determinar si la exposición repetida a ejercicio de alta intensidad con un ETM proporciona beneficios similares a los del entrenamiento en altitud.

En cuanto al tipo de mascarillas, la Organización Mundial de la Salud (OMS) (2020) hace hincapié en que deben tenerse en cuenta las siguientes características: número de capas de tela, transpirabilidad del material, cualidades hidrófobas, forma y ajuste de la mascarilla, ya que la resistencia a la respiración puede estar determinada por el grosor del material, tamaño de la mascarilla y por la cobertura total o parcial de la nariz y de la boca. Por otro lado, el uso de mascarillas como protectoras de agentes microbianos, ya sean quirúrgicas, higiénicas o con filtro de partículas FFP1-3 (filtran entre el $78 \%$ y el $98 \%$ de las partículas), el uso de respiradores integrales con filtros P100 (hasta 99.2\% de protección de mucosas y articulados virales), o las N95 que retienen el 95\% de las partículas de 0,3 $\mu \mathrm{m}$ (micrómetros), siendo las recomendadas por la National Institute of Occupational Safety and Health (NIOSH) (2020), pueden verse afectadas por la humedad, ya que durante el ejercicio se crea un microclima cálido y húmedo en el que también proliferan secreciones nasales, siendo más recomendable que las mascarillas quirúrgicas o especializadas el uso de telas sintéticas que reduzcan el calor, aunque presenten una menor barrera al flujo de entrada y salida de gérmenes. Por otra parte, ante un clima contaminado por aerosoles o goticulos con carga vírica diseminada en forma de nube, el uso de mascarillas resultaría ineficiente, ya que no se protegerían las mucosas oculares con este tipo de dispersión virulenta. En base a los estudios de turbulencia (Bourouiba, 2020), aún se tiene que evaluar la integridad y la capacidad de aislamiento de las lentes oculares como mecanismos de protección ante dispersiones víricas.

En la actualidad, tras poco más de medio año desde que inició el estado de alarma en España, existe incertidumbre acerca de cuánto tiempo el SARS-COV-2 (enfermedad Covid-19) permanece en el aire cuando se estornuda o cuando se esparcen en el aire las partículas de virus en aerosol. Siguiendo la investigación de Leung et al. (2020) las mascarillas quirúrgicas pueden reducir eficazmente la emisión de partículas del virus al medio ambiente en gotitas respiratorias, pero no en aerosoles. Según la NIOSH (2020) el uso de mascarillas o respiradores dependerá de la capacidad de protección que se necesite frente a un ambiente contaminado con una determinada carga vírica.

La elasticidad de la propia mascarilla también puede reducir la capacidad antimicrobiana de la misma, una mascarilla quirúrgica de 3 capas, de las cuales la central es una capa "Melt Brown", posee una porosidad que evita el paso de micropartículas de 0,1 $\mu \mathrm{m}$, un FFP2 o N95 de 0,3 $\mu$ m y una FFP3 de 0,023 $\mu \mathrm{m}$ (Zhu, et al., 2020), por ejemplo, el tamaño del SARS-COV-2 se sitúa entre 0,06 y 0,14 $\mu$ m, en cambio, el dióxido de carbono $\left(\mathrm{CO}_{2}\right)$ tiene $0,000232 \mu \mathrm{m}$. Por tanto, el SARSCOV-2 es aproximadamente 500 veces más grande que una molécula de $\mathrm{CO}_{2}$. Sin embargo, si la mascarilla se arruga o estira durante el ejercicio, dicha porosidad se puede ver afectada, de modo que su capacidad protectora antimicrobiana podría verse deteriorada durante el ejercicio. Por otra parte, el comportamiento de las partículas con un tamaño menor a $0,3 \mu \mathrm{m}$ es menos 
predecible que el de las partículas de gran tamaño, este comportamiento se puede describir a través del movimiento browniano, fenómeno por el cual partículas con esta masa son lo suficientemente pequeñas como para no tener impedimentos cuando viajan en el aire, describiendo un patrón errático cuando interactúan con otras moléculas en el aire como nitrógeno u oxígeno, siendo más difíciles de capturar por los filtros de las mascarillas (Del Río Haza, López, Tobón, Leyva, \& García (2020). Siguiendo a Guo, y Fu (2019) las partículas en suspensión de menos de 2,5 $\mu \mathrm{m}$ (PM2,5), como el SARS-CoV-2 que mide entre 0,08 y 0,2 $\mu \mathrm{m}$, parecen ser un mejor indicador de la contaminación urbana que las que se venían utilizando hasta ahora, las PM10 (material particulado de $10 \mu \mathrm{m}$ o menos de diámetro), esto es debido a que su origen es antropogénico en una alta proporción, es decir, los contaminantes son el resultado de actividades humanas.

Según Guo y Fu (2019) la exposición a largo plazo a material particulado de 2,5 $\mathrm{mm}$ o menos de diámetro (PM2,5), de menos de $10 \mu \mathrm{m}$ (PM10), dióxido de azufre $\left(\mathrm{SO}_{2}\right)$, dióxido de nitrógeno $\left(\mathrm{NO}_{2}\right)$, monóxido de carbono (CO) y ozono $\left(\mathrm{O}_{3}\right)$ puede provocar enfermedades cardiopulmonares. Siguiendo a Chen et al (2013) dicha exposición podría provocar infecciones respiratorias y otras comorbilidades. En la misma línea, la contaminación del aire también puede reducir capacidad cognitiva, aumenta la ansiedad y tiene otros efectos psicológicos negativos (Pun, \& Manjouride, 2017). Además, la literatura sobre salud deportiva también proporciona evidencia sobre el efecto negativo de la contaminación del aire en la salud y en el rendimiento de los atletas (Rundell, 2012).

La presente revisión analiza la literatura existente sobre el uso de la mascarilla en el ámbito deportivo en las principales bases de datos JCR y SCOPUS. Se plantean las siguientes preguntas: ¿es seguro para los deportistas hacer ejercicio físico con mascarilla?, ¿el uso de mascarillas durante el ejercicio físico afecta al rendimiento? Los 10 artículos analizados en los resultados de la revisión estudian el uso de la mascarilla y su repercusión en diferentes factores fisiológicos mientras se hace ejercicio físico. Se discute sobre las condiciones de hipoxia e hipercapnia y sobre el uso de las mascarillas para la mejora del rendimiento físico en deportistas.

\section{Método}

La revisión es integradora con un enfoque sistemático centrado en el movimiento metodológico de la Práctica Basada en la Evidencia (PBE) (Whittemore, Chao,
Jang, Minges, \& Park, 2014). Tiene un carácter cualicuantitativo, cuya principal técnica de recolección de datos fue el análisis de contenido de cada uno de los registros seleccionados de forma aleatoria, que hicieron parte de la muestra estratificada aplicada para tal fin. Este tipo de revisión integradora se centra fundamentalmente en sintetizar los conocimientos teóricos y evidencias científicas sobre las repercusiones que tiene el uso de la mascarilla en el ámbito deportivo. Esta revisión siguió las directrices de la declaración PRISMA para revisiones sistemáticas con la finalidad de estructurar el correcto desarrollo del manuscrito (Hutton et al., 2015).

La población de estudio se centró en todas aquellas publicaciones Open Access, registradas e identificadas en ISI Web of Science (WoS) y en SCOPUS, con los términos de búsqueda en sus títulos, resúmenes o palabras claves: mask y sport. Estas palabras fueron combinadas de la siguiente forma: mask AND sport. El uso de estos términos en inglés se hizo en vista que es el idioma en el que todos los trabajos indexados en WoS cuentan con registros. Lo que reduce la omisión involuntaria de casos sensibles de análisis. Por otra parte, se limitó la búsqueda a los últimos 5 años (desde enero de 2016 hasta agosto de 2020) por considerarse contribución científica reciente y debido tanto al exceso de referencias en los últimos 10 años sobre literatura que empleaba el uso de mascarillas para mejorar el rendimiento del deportista como por el silencio documental en los últimos años sobre el uso de mascarillas como protección ante un ambiente contaminado, literatura que ha proliferado en el año 2020 a causa de la pandemia.

La centralidad del estudio en torno a WoS fue motivada a que es una de las bases de datos con el mayor número de revistas y reconocimiento científico en la actualidad, además de ser la más antigua y de mayor impacto académico reconocido a nivel internacional, siendo 24891 revistas registradas si se tienen en cuenta las revisas Core de Web of Science, en contraposición a Scopus, la cual le sigue con 24701 revistas registradas. En total, se identificaron 137 registros en WoS y 118 en SCOPUS. De este total, se hizo una selección aleatoria de 34 registros en WOS y de 30 en SCOPUS, distribuidos de forma estratificada, con el 95\% de confianza y +/- 4,3 de error muestral (tabla 1). Esta selección se hizo partiendo de una tabla de números aleatoria creada ad-hoc por el autor, la cual sirvió para seleccionar cada caso de estudio por año asignando a estos un número único de identificación (véase tabla 1).

Se realizó otra búsqueda desde enero de 2016 hasta agosto de 2020 en Scholar Google, un buscador espe- 
Tabla 1. Selección aleatoria y estratificada de registros en WOS y SCOPUS.

\begin{tabular}{|c|c|c|c|c|c|c|}
\hline \multirow[t]{2}{*}{ Año } & \multicolumn{2}{|c|}{ Artículos } & \multicolumn{2}{|c|}{ Proporción } & \multicolumn{2}{|c|}{ Muestra del estrato } \\
\hline & wos & SCOPUS & wos & SCOPUS & wos & SCOPUS \\
\hline 2020 & 13 & 19 & $9,6 \%$ & $16,1 \%$ & 4 & 5 \\
\hline 2019 & 39 & 29 & $28,5 \%$ & $24,6 \%$ & 8 & 7 \\
\hline 2018 & 31 & 24 & $22,6 \%$ & $20,3 \%$ & 10 & 4 \\
\hline 2017 & 30 & 23 & $21,8 \%$ & $19,5 \%$ & 7 & 8 \\
\hline 2016 & 24 & 23 & $17,5 \%$ & $19,5 \%$ & 5 & 6 \\
\hline
\end{tabular}

Fuente: Elaborada por el autor a partir de los datos extraídos en WoS y SCOPUS.

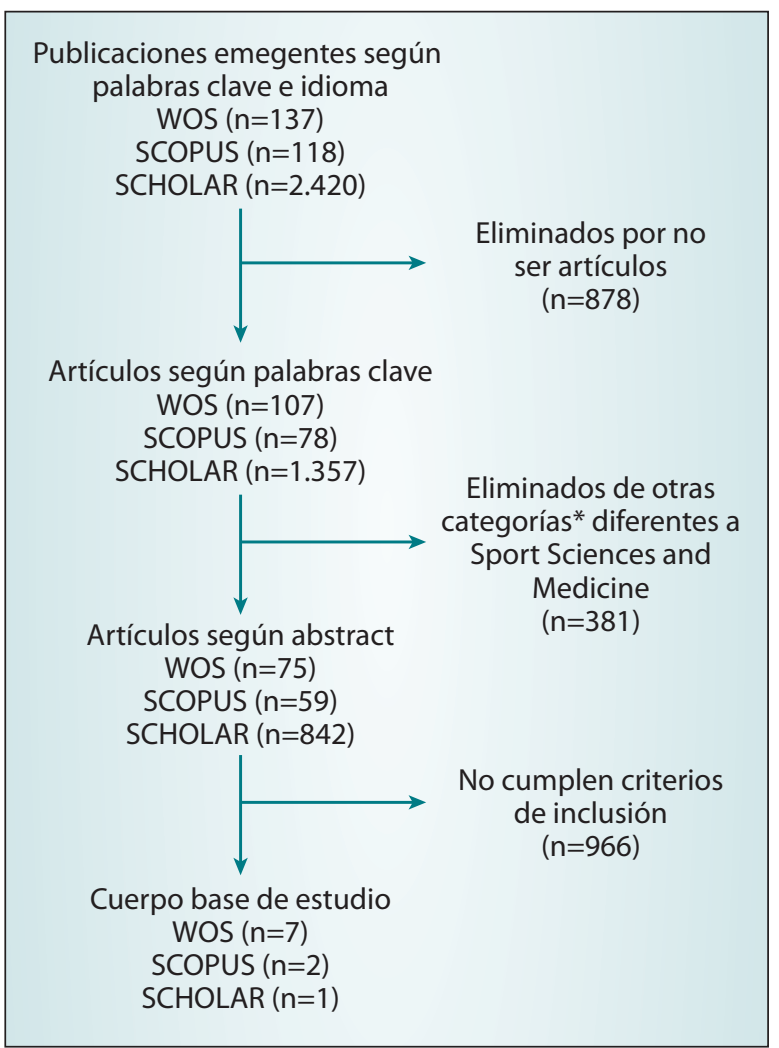

Figura 1. Flujograma de la selección del cuerpo base de estudio.

cializado en la búsqueda de contenido y bibliografía científico-académica. Las palabras clave utilizadas para la búsqueda en Scholar Google fueron: mask AND sport. Del subtotal de registros en Scholar sin incluir citas ni patentes (2420), WOS (137) y SCOPUS (118), se incluyeron 10 estudios en la revisión integradora en la última fase de inclusión de artículos, como se puede observar en el flujograma de la figura 1.

Se aplicó el referente PICR (Participantes / Intervención / Comparación / Resultados) y los siguientes criterios de inclusión: estudios peer-reviwed y Open Acces con acceso full-text, experimentales, aleatorizados y controlados con grupo experimental (con mascarilla) y control (sin mascarilla); se reflejan los diferentes parámetros fisiológicos de medida y los resultados se han obtenido con escalas y unidades de medición que han sido proporcionadas por los estudios; trabajos publicados del año 2015 en adelante, en todos los contextos geográficos, y escritos en español y en inglés; artículos pertenecientes eminentemente a las categorías* de Sport sciences, y en menor medida a las categorías Pharmacology Pharmacy, Chemistry Analytical, Biochemical Research Methods y Enginnering Electrical Electronic. Los participantes de los estudios analizados en la revisión fueron deportistas (hombres y mujeres moderadamente entrenados). En cuanto al criterio de intervención, se seleccionaron estudios experimentales y se descartaron estudios epidemiológicos y revisiones. En cuanto al criterio de comparación, se tuvieron en cuenta los estudios que incluyeron los que determinaban dos grupos, el grupo de deportistas que usaba mascarilla y pasaba por algún proceso de entrenamiento o prueba de esfuerzo, y el grupo control que no lo experimentaba. Por último, bajo el criterio de resultados, se tuvieron en cuenta aquellos estudios que demostraban estadísticamente la influencia del uso o no de la mascarilla y sus repercusiones sobre distintos parámetros fisiológicos.

Respecto a los criterios de exclusión, se han omitido comunicaciones de congresos publicados en libro de actas, trabajos de fuentes académicas no confiables, referencias anteriores al 2015, trabajos de repositorios institucionales y publicaciones en revistas de divulgación, revisiones o tesis. Por otra parte, el rigor metodológico se evaluó mediante un sistema de clasificación estructurado llamado PEDro Scale (Verhagen et al. 1998) utilizado para evaluar cuantitativamente la validez externa, la calidad metodológica y la descripción estadística de estudios aleatorizados experimentales.

Por otra parte, los niveles de evidencia, validez interna y grados de recomendación se evaluaron a través del Canadian Task Force on Preventive Health Care (CTFPHC) (2012). Por último, la probabilidad de sesgos y la preocupación sobre la aplicabilidad de los resultados se evaluó con el instrumento Quadas-2 (Ciapponi, 2015). 


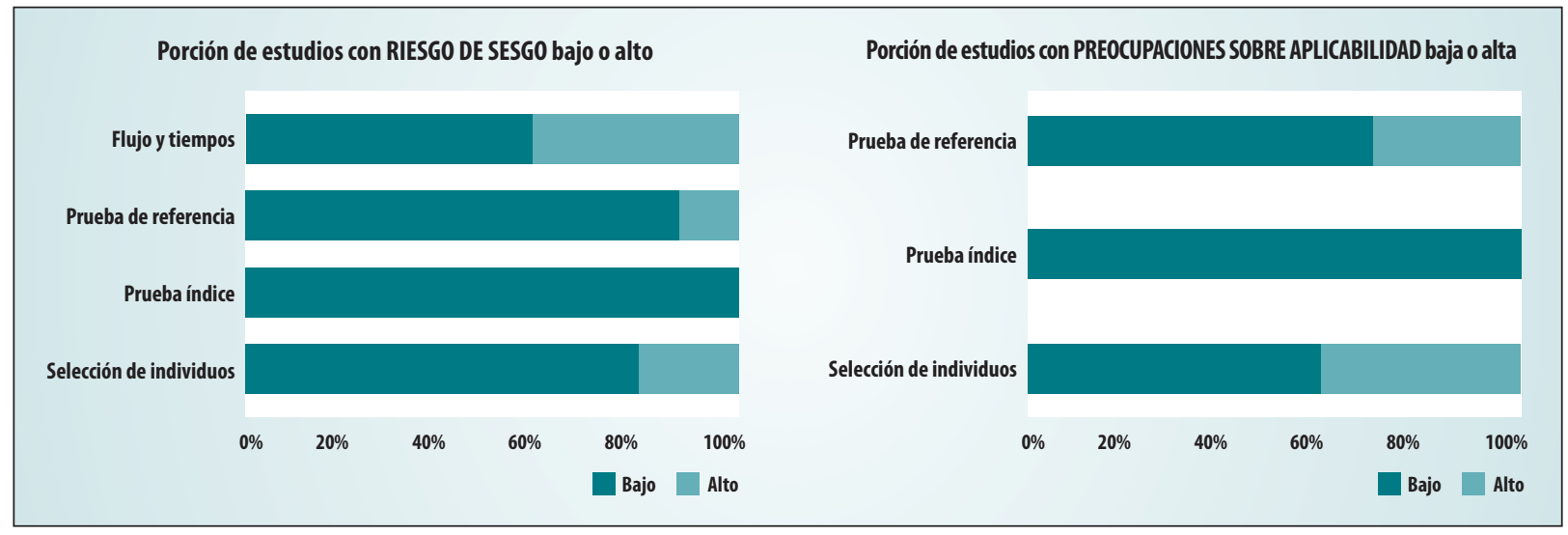

Figura 2. Estudios con riesgo de sesgo y con preocupaciones sobre aplicabilidad baja o alta.

\section{Resultados}

De los 10 artículos analizados, 7 de ellos se encuentran publicados en revistas JCR. El H index y el cuartil de cada revista fue obtenido a través del Ranking Scimago de 2019 en la categoría Sports Science. El $40 \%$ de los artículos pertenece a revistas situadas en el cuartil Q1, el 30\% en Q3, el 20\% en Q2 y el 10\% en Q4. Siguiendo el instrumento QUADAS-2 para la evaluación de la calidad de estudios de precisión diagnóstica, todos los estudios analizados en la revisión poseen una baja probabilidad de sesgo y generan poca preocupación respecto de su aplicabilidad (véase figura 2).

Respecto a PEDro Scale, la escala de 11 criterios PEDro identifica con rapidez cuales de los ensayos aleatorios pueden tener suficiente validez interna (criterios 2-9), validez externa (criterio 1: información especificada o no sobre la elección de los sujetos) y suficiente información estadística para hacer que los resultados sean interpretables (criterios 10 y 11). El 40\% de los artículos cumple con 5 o menos criterios, el $40 \%$ cumple entre 7 u 8 criterios y el $20 \% 9$ o más criterios.

En todos los estudios se les aplicó consentimiento informado a todos los participantes. El 80\% de los estudios posee validez interna y externa, poseen buena evidencia para recomendación y los resultados son fácilmente interpretables en la mayoría de ellos. No obstante, la mayoría de los estudios con grupo único, así como los estudios que comparaban grupo de control (sin mascarilla) con grupo experimental (con mascarilla), no cegaron ni a los participantes ni a los evaluadores, además, el efecto de enmascaramiento no tuvo lugar en buena parte de los estudios analizados puesto que la asignación grupal no fue oculta, por lo que se disminuyó la validez interna en este sentido y se redujo la cantidad de criterios cumplidos en PEDro Scale. Los datos extraídos de la síntesis se insertaron en la tabla 2.

\section{Discusión}

La presente revisión analiza la contribución científica sobre el uso de la mascarilla en el ámbito deportivo, planteando cuáles podrían ser sus repercusiones mientras se hace ejercicio físico. Se ha planteado si es seguro para los deportistas hacer ejercicio físico con mascarillas. En este sentido, la literatura que ha estudiado las condiciones de hipoxia e hipercapnia usando mascarilla durante el ejercicio físico es reducida y contemporánea (Jung, 2019; Pifarré, 2020; Tobin, Costalat, \& Renshaw, 2020; Wong, 2020). Siguiendo a Jung, et al. (2019) se observó en el ensayo con mascarilla una mayor respuesta en la presión arterial sistólica en reposo y una mayor frecuencia cardiaca, mientras que la saturación de la oximetría de pulso fue menor durante la prueba de ciclismo con una intensidad al $70 \%$ del $\mathrm{VO}_{2}$ pico en comparación con el ensayo sin mascarilla, concluyendo que el ciclismo de alta intensidad induce una hipoxemia moderada, retrasando la recuperación cardíaca autónoma del ejercicio. En la misma línea, en el estudio de Pifarré et al. (2020) donde evaluaron el impacto fisiológico de la hipercapnia y la hipoxia generada por las mascarillas durante la práctica de deportes aeróbicos, registraron una disminución del $\mathrm{O}_{2}$ (hipoxia) y un aumento del $\mathrm{CO}_{2}$ (hipercapnia) antes, durante y después del ejercicio, destacando una disminución en la saturación arterial de $\mathrm{O}_{2}$ post-ejercicio. Los autores concluyeron que el uso de mascarillas durante un breve ejercicio con intensidad alrededor de 6-8 METS, disminuye el $\mathrm{O}_{2}$ en un $3.7 \%$ y aumenta la concentración del $\mathrm{CO}_{2}$, aunque no fue un análisis ergo-espirométrico, sino un análisis sobre la composición gaseosa que inhaló la población objeto de estudio durante el ejercicio con mascarilla. Probablemente, durante el ejercicio físico moderado-alto la velocidad del aire inspirado y espirado contra la mascarilla es mayor, por lo que la mascarilla podría ensanchar sus poros. 
Tabla 2. Síntesis de resultados de la revisión integradora.

\begin{tabular}{|c|c|c|c|c|c|}
\hline Estudio & Rigor metodológico & Objetivo & Participantes & Procedimiento & Parámetros de medida \\
\hline $\begin{array}{l}\text { 1- Wagner, D.R., y } \\
\text { Clark, N.W. (2016). } \\
\text { Similar results for } \\
\text { face mask versus } \\
\text { mouthpiece during } \\
\text { incremental exercise } \\
\text { to exhaustion. }\end{array}$ & $\begin{array}{l}\text { - PEDro Scale: } 7 \\
\text { - 'Recomendación: A } \\
\text { - Nivel Evidencia: I } \\
\text { - Validez interna: B } \\
\text { - Validez externa: Si } \\
\text { - Citas en Scholar: } 7 \\
\text { - Revista: Journal of } \\
\text { Sports Sciences } \\
\text { - H index: } 128 \text { (Q1) }\end{array}$ & $\begin{array}{l}\text { Comparar los datos } \\
\text { de análisis de gases } \\
\text { metabólicos entre } \\
\text { boquilla con clip } \\
\text { nasal y mascarilla. }\end{array}$ & $\begin{array}{l}14 \text { voluntarios, } 7 \\
\text { hombres y } 7 \text { mujeres } \\
\text { (25,1 } \pm 2,7 \text { años). }\end{array}$ & $\begin{array}{l}\text { Completaron dos } \\
\text { pruebas máximas } \\
\text { aleatorias en cinta } \\
\text { rodante en } 1 \text { semana, } \\
\text { una prueba con clip } \\
\text { nasal y otra con } \\
\text { mascarilla. }\end{array}$ & $\begin{array}{l}\text { - Volumen Máx. de Oxígeno } \\
\text { (VO,max) } \\
\text { - Ventilación por minuto }(\mathrm{VM}) \\
\left.\text { - Fracción de } \mathrm{O}_{2} \text { espirado }(\mathrm{FEO})_{2}\right) \\
\left.\text { - Dióxido de carbono }(\mathrm{FECCO})_{2}\right) \\
\text { - Frecuencia respiratoria (FR) } \\
\text { - Volumen corriente }(\mathrm{VC}) \\
\text { - Frecuencia Cardiaca }(\mathrm{FC}) \\
\text { - Esfuerzo percibido (EP) }\end{array}$ \\
\hline
\end{tabular}

Resultados y conclusiones

La diferencia en el consumo máximo de oxígeno ( $\mathrm{VO}$ max) entre clip nasal $(52,7 \pm 11,3 \mathrm{ml} \cdot \mathrm{kg}-1$. min-1) y mascarilla $(52,2 \pm 11,7$ $\mathrm{min}-1)$ y mascarilla $(52,2 \pm 11,7$
$\mathrm{ml} \cdot \mathrm{kg}-1 \cdot \mathrm{min}-1)$ no fue significativa $(p=, 53)$. Además, las diferencias entr clip nasal y mascarilla en el resto de parámetros no fue significativo $(p>, 05)$. La selección de clip nasal o mascarilla no afecta en el intercambio de gases ni en los patrones de respiración del participante.

\begin{tabular}{|c|c|}
\hline $\begin{array}{l}\text { 2- Porcari, J.P. et } \\
\text { al (2016). Effect of } \\
\text { wearing the elevation } \\
\text { training mask on } \\
\text { aerobic capacity, } \\
\text { lung function, and } \\
\text { hematological. } \\
\text { variables. }\end{array}$ & $\begin{array}{l}\text { - PEDro Scale: } 10 \\
\text { - oRecomendación: A } \\
\text { - Nivel Evidencia: I } \\
\text { - Validez interna: B } \\
\text { - Validez externa: Si } \\
\text { - Citas en Scholar: } 51 \\
\text { - Revista: Journal of } \\
\text { Sports Science and } \\
\text { Medicine } \\
\text { - H index: } 58 \text { (Q2) }\end{array}$ \\
\hline
\end{tabular}

Conocer los efectos
de la Elevation
Training Mask 2.0
(ETM), que simula
supuestamente el
entrenamiento en
altitud.

$\begin{array}{lll}25 \text { voluntarios } & \text { Completaron } & \text { - Volumen Máx. de Oxígeno } \\ \text { (16 hombres } & 6 \text { semanas de } & \text { (VO2max) } \\ \text { y 9 mujeres) } & \text { entrenamiento en } & \text { - Umbral ventilatorio (UV) } \\ \text { moderadamente } & \text { cicloergómetro de alta } & \text { - Umbral de Compensación } \\ \text { entrenados. } & \text { intensidad, el grupo } & \text { Respiratoria (UCR) } \\ & \text { control sin mascarilla } & \text { - Función pulmonar } \\ & \text { (12) y experimental } & \text { - Presión de inspiración máxima } \\ & \text { con mascarilla (12) se } & \text { - Hemoglobina } \\ & \text { asignó al azar. } & \text { - Hematocrito }\end{array}$

Hubo una mejora significativa en el $\mathrm{VO}_{2}$ máx tanto en el grupo de control $(13,5 \%)$ como en el de mascarilla $(16,5 \%)$. Solo el grupo de mascarilla tuvo mejoras significativas en el umbral tuvo mejoras significativas en el umbral
ventilatorio (UV) $(13,9 \%)$, producción de potencia (PO) en UV (19,3\%), umbral de potencia (PO) en UV (19,3\%), umbación respiratoria (UCR) $(10,2 \%)$ y PO en el UCR $(16,4 \%)$ de antes y después de la prueba. Usar el ETM no parece actuar como un simulador de altitud, sino más como un dispositivo de entrenamiento de los músculos respiratorios.

\begin{tabular}{ll}
\hline 3- Sellers, J.H., & - PEDro Scale: 8 \\
Monaghan, T.P., & - ORecomendación: A \\
Schnaiter, J.A., & - Nivel Evidencia: I \\
Jacobson, B.H., y Pope, & - Validez interna: B \\
Z.K. (2016). Efficacy & - Validez externa: 5 si \\
of a Ventilatory & - Citas en Scholar: 11 \\
Training Mask to & - Revista: Journal \\
Improve Anaerobic & of Strength and \\
and Aerobic Capacity & Conditioning Research \\
in Reserve Officers' & - H index: 121 (Q1) \\
Training Corps Cadets. & \\
\hline
\end{tabular}

\begin{tabular}{|c|c|c|c|}
\hline $\begin{array}{l}\text { Examinar la eficacia } \\
\text { de una mascarilla } \\
\text { de entrenamiento } \\
\text { ventilatorio para } \\
\text { mejorar la aptitud } \\
\text { anaeróbica/ aeróbica } \\
\text { en cadetes. }\end{array}$ & $\begin{array}{l}17 \text { cadetes } \\
\text { del Cuerpo de } \\
\text { Entrenamiento de } \\
\text { Oficiales de Reserva } \\
\text { (CEOR) de una } \\
\text { universidad del } \\
\text { Medio Oeste. }\end{array}$ & $\begin{array}{l}\text { Entrenamiento físico } \\
\text { de } 6 \text { semanas, el } \\
\text { grupo control sin } \\
\text { mascarilla (8) y grupo } \\
\text { experimental con } \\
\text { mascarilla (9) con } \\
\text { ajuste para simular } \\
\text { una altitud de } 2750 \mathrm{~m} \text {. }\end{array}$ & $\begin{array}{l}\text { - Volumen Máx. de Oxígeno } \\
\text { (VO max) } \\
\text { - Antropometría } \\
\text { - Prueba anaeróbica de Wingate } \\
\text { de } 30 \text { (WAnT) } \\
\text { - Índice de fatiga. }\end{array}$ \\
\hline
\end{tabular}

No hubo un efecto significativo $(\mathrm{p} \leq$ 0.05 ) entre los grupos sobre el índice de fatiga, la capacidad anaeróbica, la potencia máxima, el VO máx. o en el tiempo hasta el agotamiento. Estos resultados sugieren que el uso de la máscara de entrenamiento ventilatorio durante el entrenamiento no provocó adaptaciones aeróbicas o anaeróbicas superiores en los cadetes del CEOR.

\begin{tabular}{lllll}
\hline 4- Guo, M., y Fu, S. & - PEDro Scale: 4 & Relacionar el nivel & 314.341 corredores & Los datos del índice \\
(2019). Running with & - oRecomendación: M & de contaminación & populares de & diario de calidad del \\
a Mask? The Effect & - Nivel Evidencia: II-2 & en el aire con el & maratón en 37 & aire (IDCA) proceden \\
of Air Pollution on & - Validez interna: M & rendimiento de & países en 56 eventos & del Ministerio de \\
Marathon Runners' & - Validez externa: $\mathrm{Si}$ & los corredores a & de carreras en China & Protección Ambiental \\
Performance. & - Citas en Scholar: 7 & través de un modelo & entre 2014 y 2015. & de China y los datos \\
& - Revista: Journal of & matemático. & & meteorológicos de \\
& Sports Economics & & la base de datos de \\
& - H index: 44 (Q3) & & & Bloomberg.
\end{tabular}

El estándar establecido por la Organización Mundial de la Salud (OMS) para las concentraciones de PM2.5, PM10 y S02 es de 25, 50 y $20 \mathrm{ug} / \mathrm{m} 3$ de media cada 24 horas, respectivamente. Sin embargo, las medias durante las horas de carrera en la muestra de estudio fueron aproximadamente de 74, 105 y 22 ug/

\section{-Indices de 6 contaminantes: partículas en suspensión de $-2,5 \mu \mathrm{m}$ (PM2.5), de $-10 \mu \mathrm{m}$ (PM10), dióxido de azufre (SO2)
dióxido de nitrógeno (NO2) dióxido de nitrógeno (NO2),
monóxido de carbono (CO) y ozono (03) \\ -Fórmula matemática: índice de polución, condiciones climáticas, temperatura, velocidad del viento, humedad relativa y precipitación, filtrando por edad y género.}

\begin{tabular}{ll}
\hline 5- Jung, H.C., Lee, & - PEDro Scale: 8 \\
N.H., John, S.D., y & - ${ }^{\circ}$ Recomendación: A \\
Lee, S. (2019). The & - Nivel Evidencia: I \\
elevation training & - Validez interna: B \\
mask induces modest & - Validez externa: si \\
hypoxaemia but does & - Citas en Scholar: 3 \\
not affect heart rate & - Revista: Biology of \\
variability during & Sport \\
cycling in healthy & - H index: 23 (Q2) \\
adults. &
\end{tabular}

\begin{tabular}{|c|c|}
\hline $\begin{array}{l}\text { Examinar los efectos } \\
\text { de la mascarilla } \\
\text { de entrenamiento } \\
\text { de elevación } \\
\text { (ETM) sobre la } \\
\text { hemodinámica y la } \\
\text { FC en ciclistas sanos. }\end{array}$ & $\begin{array}{l}20 \text { voluntarios } \\
\text { iniciales, pero } \\
\text { incluidos } 15 \text { ciclistas } \\
\text { sanos ( } 9 \text { hombres y } \\
6 \text { mujeres con } 27,0 \\
\pm 1,14 \text { años) como } \\
\text { criterios de elección: } \\
\% \text { grasa }-30 \% \text { y sin } \\
\text { patologías. }\end{array}$ \\
\hline
\end{tabular}

Completaron 2 - Presión arterial entrenamientos de - Frecuencia Cardíaca (FC) entrenamientos de con y sin mascarilla, con 3 fases de 10 $50 \%$ de $\mathrm{VO}_{2}$ pico, $70 \%$ de Vo, 2 pico y recuperación. Se utilizó un monitor ECG Actiwave-Cardio para medir las variables. Saturación de la oximetría de pulso $\left(\mathrm{SPO}_{2}\right)$ en cada fase $\mathrm{m} 3$, lo que sugiere efectos nocivos sobre el rendimiento de los corredores.

Se observó una mayor respuesta en la presión arterial sistólica $(\mathrm{p}=.035)$ en reposo, una mayor frecuencia cardíaca $(p=.047)$, un menor intervalo entre latidos durante la recuperación $(\mathrm{p}=.01)$ y una menor $\mathrm{SPO}_{2}(\mathrm{p}=.033)$ durante $\mathrm{e}$ ciclismo de alta intensidad (70\% del $\mathrm{VO}_{2}$ pico) en el ensayo con mascarilla. El uso de un ETM durante el ciclismo de alta intensidad (70\% del VO pico) induce una hipoxemia moderada, retrasando la recuperación cardíaca autónoma del ejercicio.

\begin{tabular}{|c|c|c|c|c|c|c|}
\hline $\begin{array}{l}\text { 6- Bellovary, B.N. et } \\
\text { al. (2019). Effects of } \\
\text { high-intensity interval } \\
\text { training while using a } \\
\text { breathing-restrictive } \\
\text { mask compared to } \\
\text { intermittent hypobaric } \\
\text { hypoxia. }\end{array}$ & $\begin{array}{l}\text { - PEDro Scale: } 8 \\
\text { - oRecomendación: A } \\
\text { - Nivel Evidencia: I } \\
\text { - Validez interna: B } \\
\text { - Validez externa: Sí } \\
\text { - Citas en Scholar: } 2 \\
\text { - Revista: Journal of } \\
\text { Human Sport and } \\
\text { Exercise } \\
\text { - H index: } 23 \text { (Q3) }\end{array}$ & $\begin{array}{l}\text { Determinar los } \\
\text { efectos de la ETM } \\
\text { en comparación con } \\
\text { el entrenamiento } \\
\text { hipóxico intermitente } \\
\text { (IHT) sobre el } \\
\text { rendimiento aeróbico } \\
\text { y la economía en } \\
\text { ciclismo. }\end{array}$ & $\begin{array}{l}30 \text { ciclistas } \\
\text { voluntarios sin } \\
\text { hipertensión ni } \\
\text { enfermedades } \\
\text { previas (15 hombres } \\
\text { y } 15 \text { mujeres) } \\
\text { asignados al azar en } \\
3 \text { grupos: ETM (10), } \\
\text { IHT (10) y grupo } \\
\text { control (10). }\end{array}$ & $\begin{array}{l}\text { Realizaron dos } \\
\text { pruebas/semana de } 20^{\circ} \\
\text { de HIIT en } 6 \text { semanas } \\
\text { midiendo V0 } 0_{2} \text { máx en } \\
\text { cicloergómetro con } \\
\text { recuperación activa } \\
\text { de } 90^{\prime \prime} \text { a } 25 \mathrm{~W}(10 \\
\text { episodios) con entrada } \\
\text { y salida de } 5^{\prime} \text {. }\end{array}$ & $\begin{array}{l}\text { - Volumen Máx. de Oxígeno } \\
\text { (VO }{ }_{2} \text { max) } \\
\text { - Potencia submáxima (PO) de } \\
100,125 \text { y } 150 \mathrm{~W}\end{array}$ & $\begin{array}{l}\text { Todos los participantes mejoraron el } \\
\text { VO max y PO en el umbral ventilatorio } \\
\text { antes y después del entrenamiento. } \\
\text { Los grupos ETM e IHT respondieron de } \\
\text { manera similar en el esfuerzo máximo } \\
\text { y submáximo después de seis semanas } \\
\text { de entrenamiento. El grupo IHT, } \\
\text { pero no el grupo ETM, experimentó } \\
\text { un aumento de energía glucolítica } \\
\text { durante el ejercicio submáximo. }\end{array}$ \\
\hline $\begin{array}{l}\text { 7- Freemas, J.A., } \\
\text { Wilhite, D.P, } \\
\text { Greenshields, J.T., } \\
\text { Adamic, E.M., y } \\
\text { Mickleborough, T.D. } \\
\text { (2020). Comparison } \\
\text { between a facemask } \\
\text { and mouthpiece on } \\
\text { breathing mechanics } \\
\text { and gas exchange } \\
\text { variables during high- } \\
\text { intensity exercise. }\end{array}$ & $\begin{array}{l}\text { - PEDro Scale: } 9 \\
\text { - oRecomendación: A } \\
\text { - Nivel Evidencia: I } \\
\text { - Validez interna: B } \\
\text { - Validez externa: si } \\
\text { - Citas en Scholar: } 1 \\
\text { - Revista: European } \\
\text { Journal of Sport } \\
\text { Science } \\
\text { - H index: } 47 \text { (Q1) }\end{array}$ & $\begin{array}{l}\text { Comparar } \\
\text { la mecánica } \\
\text { respiratoria, el } \\
\text { intercambio de gases } \\
\text { y la disnea percibida } \\
\text { (RPB) durante el } \\
\text { ejercicio de alta } \\
\text { intensidad entre } \\
\text { una boquilla y una } \\
\text { mascarilla facial. }\end{array}$ & $\begin{array}{l}14 \text { hombres } \\
\text { entrenados } 7 \text { con } \\
\text { mascarilla y } 7 \text { con } \\
\text { boquilla) con función } \\
\text { pulmonar normal } \\
\text { y que alcanzan un } \\
\text { pico de VO } \mathrm{O}_{2} \text { de } 45 \\
\text { ml-kg-1·min-1 o } \\
\text { mayor. }\end{array}$ & $\begin{array}{l}\text { Ensayo aleatorizado } \\
\text { en grupo con boquilla } \\
\text { (Hans Rudolph) y } \\
\text { con una mascarilla } \\
\text { facial ( } 7450, \text { Hans } \\
\text { Rudolph) de } 6 \text { ' al } \\
90 \% \text { de } \mathrm{VO}_{2} \text { pico sobre } \\
\text { un cicloergómetro } \\
\text { (Velotron Pro, Racer } \\
\text { Mate,Inc.), }\end{array}$ & $\begin{array}{l}\text { - Mecánica respiratoria } \\
\text { - Intercambio de gases } \\
\text { - Disnea percibida (DP) }\end{array}$ & $\begin{array}{l}\text { No hubo diferencias significativas } \\
\text { en la mecánica respiratoria, las } \\
\text { variables de intercambio de gases } \\
\text { o la RPB. Estos datos sugieren que } \\
\text { la mascarilla facial se puede seguir } \\
\text { usando indistintamente con la boquilla } \\
\text { e incluso puede ser una alternativa } \\
\text { más cómoda durante el ejercicio de } \\
\text { alta intensidad. }\end{array}$ \\
\hline
\end{tabular}


Tabla 2. Síntesis de resultados de la revisión integradora (continuación).

\begin{tabular}{|c|c|c|c|c|c|c|}
\hline Estudio & Rigor metodológico & Objetivo & Participantes & Procedimiento & Parámetros de medida & Resultados y conclusiones \\
\hline $\begin{array}{l}\text { 8-Pifarré, F., Zabala, } \\
\text { D.D., Grazioli, G., y } \\
\text { Maura, I. D. (2020). } \\
\text { Covid-19 and mask } \\
\text { in sports. }\end{array}$ & $\begin{array}{l}\text { - PEDro Scale: } 5 \\
\text { - 'Recomendación: B } \\
\text { - Nivel Evidencia: II-1 } \\
\text { - Validez interna: B } \\
\text { - Validez externa: no } \\
\text { - Citas en Scholar: } 3 \\
\text { - Revista: Apunts } \\
\text { Medicina de l'Esport } \\
\text { - H index: } 10 \text { (Q4) }\end{array}$ & $\begin{array}{l}\text { Evaluar el impacto } \\
\text { fisiológico de la } \\
\text { hipercapnia y la } \\
\text { hipoxia generada } \\
\text { por las mascarillas } \\
\text { durante la práctica } \\
\text { de deportes } \\
\text { aeróbicos. }\end{array}$ & $\begin{array}{l}8 \text { sujetos ( } 6 \text { hombres } \\
\text { y } 2 \text { mujeres) }\end{array}$ & $\begin{array}{l}\text { Protocolo de Ruffier } \\
\text { mediante } 21 \text { flexiones } \\
\text { entre 6-8 METS, con } \\
\text { y sin mascarilla, al } \\
\text { aire libre. Se utilizó un } \\
\text { pulsioxímetro (FS20C } \\
\text { TEMPI-TEC) y un } \\
\text { analizador de gases } \\
\text { (MultiRae de Rae } \\
\text { Systems@). }\end{array}$ & $\begin{array}{l}\text { - Frecuencia cardíaca }(\mathrm{FC}) \text {. } \\
\text { - Concentración de } \mathrm{O}_{2} \text { y CO} \\
\text { dentro de la mascarilla. } \\
\text { - Saturación arterial de } \mathrm{O}_{2} \\
\left(\mathrm{SatO}_{2}\right) \text {. }\end{array}$ & $\begin{array}{l}\text { Se registró una disminución del } \mathrm{O}_{2} \\
\text { antes del ejercicio }(20,9 \%) \text {, durante } \\
(18,3 \%) \text { y post-ejercicio }(17,8 \%) \\
(\mathrm{p}<, 001) \text {, un aumento del } \mathrm{CO}_{2} \text { en las } \\
\text { tres condiciones previas }(464,14162, \\
17000 \mathrm{ppm}, \text { respectivamente; } p<, 001) \\
\text { y una disminución de la saturación } \\
\text { basal de } \mathrm{O}_{2}(97,6 \pm 1,5 \%) \text { respecto a la } \\
\text { saturación arterial de } \mathrm{O}_{2} \text { post-ejercicio } \\
(92,1 \pm 4,12 \%) \text { ( }<<, 02) \text {. El uso de } \\
\text { mascarillas en deportistas disminuye } \\
\text { el } O_{2} \text { en un } 3.7 \% \text {, provoca respiración } \\
\text { hipóxica e hipercápnica en un esfuerzo } \\
\text { de 6-8 METS. }\end{array}$ \\
\hline $\begin{array}{l}\text { 9-Tobin, B., Costalat, } \\
\text { G., y Renshaw, G.M.C. } \\
\text { (2020). Intermittent } \\
\text { not continuous } \\
\text { hypoxia provoked } \\
\text { haematological } \\
\text { adaptations in healthy } \\
\text { seniors: hypoxic } \\
\text { pattern may hold } \\
\text { the key. }\end{array}$ & 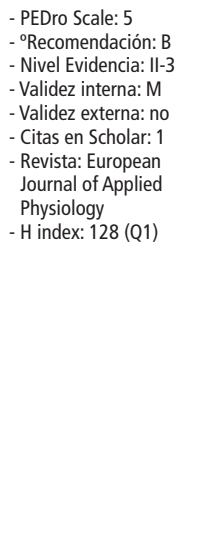 & $\begin{array}{l}\text { Investigar el efecto } \\
\text { de dos patrones } \\
\text { hipóxicos (continuos } \\
\text { e intermitentes) } \\
\text { sobre la adaptación } \\
\text { hematológica, } \\
\text { el estrés y el } \\
\text { daño cardíaco } \\
\text { en participantes } \\
\text { mayores sanos }\end{array}$ & $\begin{array}{l}15 \text { participantes } \\
\text { mayores sin } \\
\text { enfermedad previa }\end{array}$ & $\begin{array}{l}\text { Protocolo de tres fases } \\
\text { durante } 3 \text { semanas } \\
\text { consecutivas: } 5 \\
\text { días al aire libre sin } \\
\text { mascarilla, } 5 \text { días } \\
\text { de respiración con } \\
\text { mascarilla normóxica } \\
\left(\mathrm{FiO}_{2}=21 \%\right)^{2} \text {, y } 5 \text { días } \\
\text { de hipoxia intermitente } \\
\text { (HI) adaptados para } \\
\text { lograr una saturación } \\
\text { de oxígeno periférica } \\
\text { media (SpO }{ }_{2} \text { ) del } \\
85 \% \text { durante } ~ 70^{\prime} \text { de } \\
\text { exposición acumulada a } \\
\text { la hipoxia. Después de } \\
\text { un período de lavado } \\
\text { de } 5 \text { meses, se recordó } \\
\text { a los participantes } \\
\text { que realizaran } \\
\text { hipoxia continua (CH, } \\
\left.\mathrm{SpO}_{2}=85 \%, \sim 70^{\prime}\right) \text {. }\end{array}$ & $\begin{array}{l}\text { - Recuento de glóbulos rojos } \\
\text { (RBCc) } \\
\text { - Concentración de hemoglobina } \\
\text { ([Hb]) } \\
\text { - Hematocrito (Hct) } \\
\text { - Porcentaje de reticulocitos (\% } \\
\text { Retics) } \\
\text { - Inmunoglobulina A secretora } \\
\text { (S-IgA) } \\
\text { - Cortisol } \\
\text { - Troponina T cardíaca (cTnT) }\end{array}$ & $\begin{array}{l}\text { El RBCc solo aumentó el día } 5 \text { de } \\
\text { tratamiento con Hipoxia intermitente } \\
\text { (IH) en comparación con los valores } \\
\text { iniciales del día } 5(+7,7 \%, \mathrm{p}<, 01) \text { y los } \\
\text { valores simulados del día } 5(+12,9 \% \text {, } \\
\mathrm{p}<, 01) \text {. La } \mathrm{Hb} \text { sólo aumentó en el día } 5 \\
\text { de tratamiento con IH en comparación } \\
\text { con los valores iniciales del día } 5 \\
(+14,7 \%, \mathrm{p}<0,01) \text { y los valores } \\
\text { simulados del día } 5(+14,3 \%, \mathrm{p}<, 01) \text {. } \\
\text { No se observaron diferencias en } \mathrm{S}-\mathrm{IgA} \text {, } \\
\text { cortisol o cTnT después de IH o Hipoxia } \\
\text { Continua (CH). Estos resultados } \\
\text { revelaron que las diferencias } \\
\text { inherentes en los patrones hipóxicos } \\
\text { de IH y CH podrian proporcionar } \\
\text { componentes cruciales necesarios para } \\
\text { desencadenar cambios hematológicos } \\
\text { en personas mayores, sin provocar } \\
\text { respuestas de estrés inmunológico o } \\
\text { dañar el miocardio. }\end{array}$ \\
\hline $\begin{array}{l}\text { 10-Wong, A.Y. et al. } \\
\text { (2020). Impact of the } \\
\text { Covid-19 pandemic on } \\
\text { sports and exercise }\end{array}$ & $\begin{array}{l}\text { - PEDro Scale: } 5 \\
\text { - oRecomendación: A } \\
\text { - Nivel Evidencia: I } \\
\text { - Validez interna: B } \\
\text { - Validez externa: si } \\
\text { - Citas en Scholar: 0 } \\
\text { - Revista: Asia-Pacific } \\
\text { Journal of Sports } \\
\text { Medicine, Arthroscopy, } \\
\text { Rehabilitation and } \\
\text { Technology } \\
\text { - H index: } 8 \text { (Q3) }\end{array}$ & $\begin{array}{l}\text { Investigar el efecto } \\
\text { fisiológico de usar } \\
\text { una mascarilla } \\
\text { durante el ejercicio }\end{array}$ & $\begin{array}{l}23 \text { deportistas } \\
\text { voluntarios sanos } \\
\text { (10 hombres y } 13 \\
\text { mujeres con edad } \\
\text { media de } 33,8 \text { años) }\end{array}$ & $\begin{array}{l}\text { Se realizaron } \\
\text { caminatas escalonadas } \\
\text { en tapiz rodante a } 4 \\
\mathrm{~km} / \mathrm{h} \text { durante } 6^{\prime} \text { con y } \\
\text { sin usar una mascarilla } \\
\text { quirúrgica, y en un } \\
\text { orden aleatorio con } \\
\text { suficiente tiempo de } \\
\text { descanso entre los } \\
\text { ensayos. }\end{array}$ & $\begin{array}{l}\text {-Frecuencia Cardíaca (FC) } \\
\text {-Tasa de Esfuerzo Percibido (TEP) }\end{array}$ & $\begin{array}{l}\text { La frecuencia cardíaca de los sujetos } \\
\text { que usaban una mascarilla fue de } 128 \\
\text { latidos por minuto y la TEP de } 12,7 \\
\text { sobre } 20 \text {. En aquellos sin mascarilla, } \\
\text { los resultados fueron una frecuencia } \\
\text { cardíaca de } 124 \text { latidos por minuto y } \\
\text { un RPE de } 10,8 \text {. Usar una mascarilla } \\
\text { eleva significativamente la frecuencia } \\
\text { cardiaca y el esfuerzo percibido. }\end{array}$ \\
\hline
\end{tabular}

oRecomendación: Buena evidencia para recomendación (A) / Evidencia moderada (B) / Evidencia contradictoria (C) / Evidencia moderada para no recomendación (D) / Buena evidencia para no recomendación (E) / Evidencia insuficiente para recomendación (I)

Nivel de evidencia: con asignación aleatoria (I) / sin asignación aleatoria (II-1) / evidencia existente en estudios de controles (II-2) / evidencia existente con o sin intervención sin asignación aleatoria (II-3) / Evidencia existente a partir de la opinión de expertos (III)

Validez interna: cumple criterios espećíficos de un estudio bien diseñado (B: Buena) / no cumple al menos uno de los criterios específicos, pero sin defectos metodológicos (M: moderada) / estudio con al menos un defecto metodológico grave (I: insuficiente).

Por otro lado, la molécula de $\mathrm{CO}_{2}$ espirada, al ser tan pequeña $(0,000232 \mu \mathrm{m})$, puede traspasar cualquier mascarilla quirúrgica o con filtro de partículas. Ahora bien, durante el ejercicio físico existe una mayor frecuencia respiratoria y el aire renovado en cada respiración por unidad de tiempo es menor en comparación con un estado basal. Probablemente, con la mascarilla el ciclo respiratorio tiende a tener más duración y el esfuerzo subjetivo percibido podría ser mayor. En la misma línea, en el estudio de Wong et al. (2020) concluyeron que usar una mascarilla eleva significativamente la frecuencia cardíaca y el esfuerzo percibido tan solo caminando a $4 \mathrm{~km} / \mathrm{h}$.

En cuanto al uso de mascarillas para mejorar el rendimiento en deportistas, en esta revisión se ha planteado si el uso de mascarillas durante el ejercicio físico afecta al rendimiento. Siguiendo a Porcari et al. (2016) solo el grupo que utilizó la Elevation Training Mask (ETM) tuvo mejoras significativas en el umbral ventilatorio, producción de potencia y en el umbral de compensación respiratoria, concluyendo que el uso del ETM mientras se participa en un programa de entrenamiento de cicloergómetro intenso durante 6 semanas no parece actuar como un simulador de altitud, sino más como un dispositivo de entrenamiento de los músculos respiratorios. Por otro lado, el estudio de Sellers et al. (2016) comprobó que, entre los grupos con y sin mascarilla, no hubo diferencias sobre el índice de fatiga, la capacidad anaeróbica, la potencia máxima, el $\mathrm{VO}_{2}$ máx. o en el tiempo hasta el agotamiento, resultados que confirmaron que el uso de la mascarilla de entrenamiento ventilatorio durante el entrenamiento no provocó adaptaciones aeróbicas o anaeróbicas superiores en los cadetes del Cuerpo de Entrenamiento de Oficiales de Reserva (CEOR). Sellers et al. (2016) recomendaron que se utilicen métodos de entrenamiento en altitud 
simulados más establecidos cuando se incorpore entrenamiento hipóxico intermitente. En este sentido, la mayoría de estudios en este ámbito comparan grupos que emplean el ETM y los controles para determinar el efecto sobre el rendimiento aeróbico. En cambio, en el estudio de Bellovary et al. (2019) realizaron comparaciones con el entrenamiento hipóxico intermitente (IHT) dando a conocer cómo afecta el ETM y el IHT a la economía del ejercicio. En el estudio de Bellovary et al. (2019) determinaron los efectos sobre el rendimiento aeróbico y la economía en ciclistas mediante entrenamiento hipóxico intermitente (IHT) y ETM, los resultados mostraron que el grupo IHT, pero no el grupo ETM, experimentó un aumento de energía glucolítica durante el ejercicio submáximo, es decir, mejor rendimiento anaeróbico láctico. Por otro lado, Tobin et al. (2020) estudiaron el efecto de dos patrones hipóxicos continuos $(\mathrm{CH})$ e intermitentes $(\mathrm{IH})$ sobre la adaptación hematológica, el estrés y el daño cardíaco en participantes mayores sanos, concluyendo que el recuento de glóbulos rojos y la concentración de hemoglobina aumentaron al quinto día de tratamiento con $\mathrm{IH}$.

En la situación pandémica actual se deben establecer determinadas medidas de uso de distinto tipo de mascarilla en función del tipo e intensidad de ejercicio físico que se vaya a realizar. En el estudio de Guo, y Fu (2019) relacionaron el nivel de contaminación en el aire con el rendimiento de los corredores a través de un modelo matemático, tuvieron en cuenta los datos del índice diario de calidad del aire y de las condiciones meteorológicas a través de Bloomberg, comprobando que las concentraciones los días de la carrera en distintas ciudades de China superaban los estándares establecidos por la OMS. Se hace necesaria la investigación en la línea de los artículos analizados en esta revisión y la recopilación de información proporcionada por organismos como la OMS, NIOSH o de la UE, que ayude a frenar la propagación del SARS-COV-2 durante la práctica del ejercicio físico.

\section{Conclusiones}

Una vez realizada la revisión de la literatura, se destaca que el uso de mascarillas en deportistas provoca respiración hipóxica e hipercápnica, siendo más evidente en ejercicios de intensidad moderada a alta de más de 6 METS. Por otro lado, el uso de una mascarilla como la Elevation Training Mask (ETM) no parece actuar como un simulador de altitud, sino más bien como un dispositivo de entrenamiento de los músculos respiratorios. Además, el entrenamiento hipóxico intermitente (IHT) en altura, al contrario que la ETM, mejora el rendimiento anaeróbico láctico. Por otra par- te, no hay diferencias significativas en el intercambio de gases ni en los patrones de respiración utilizando una mascarilla o un clip nasal y boquilla en pruebas de esfuerzo, aunque, por un lado, el empleo de clip nasal y boquilla en pruebas incrementales podría alterar los patrones de respiración durante el ejercicio, y, por otro, la mascarilla, además de ser más cómoda, permite alcanzar un pico constantemente más alto de potencia. Por último, siguiendo las consideraciones de varios autores (Toresdahl, \& Asif, 2020; Wong et al., 2020) y de acuerdo a los resultados de los estudios analizados en la presente revisión integradora, no se debería recomendar a los deportistas asintomáticos que usen una mascarilla mientras realizan ejercicio físico para evitar la infección con Covid-19 en el entorno comunitario o mientras se viaja, ya que no se reduce significativamente el riesgo de infección y se aumentaría la carga fisiológica del cuerpo, especialmente en aquellos con múltiples comorbilidades subyacentes.

\section{Limitaciones y futuras líneas de investigación}

El tamaño de las muestras de la mayoría de los estudios analizados no es representativo y tampoco se proporcionan datos sobre las características de los diferentes tipos de mascarillas empleados, lo que conlleva un alto grado de subjetividad y las conclusiones podrían no ser extrapolables al estado del arte. Tampoco se muestran resultados sobre heterogeneidad entre los resultados del grupo control/experimental de los distintos estudios analizados. En esta línea, los resultados de la presente revisión deberían combinarse a través de un meta-análisis que permitiera valorar las discrepancias de heterogeneidad clínica (participantes, intervenciones, desenlaces), metodológica (diseño, realización, estrategia de análisis) y estadística (variabilidad aleatoria muestral). Por otra parte, la calidad metodológica de los estudios incluidos en el análisis de los resultados no se ha consensuado entre dos o más revisores. Aunque los estudios analizados en la revisión cumplen criterios de validez externa, poseen un alto nivel de evidencia científica y un buen grado de recomendación, echándose en falta referencias de más alto nivel indexadas en JCR en este campo de actuación.

Por otra parte, se hace necesaria la investigación en instrumental que ayude a monitorear el flujo y volumen de aire respiratorio. En esta línea, el estudio de Zhou, Costa, \& Lukowicz (2020) en el que concluyen que los barómetros con huellas milimétricas incrustadas en las mascarillas pueden funcionar de manera similar a un espirómetro digital que ayude en el monitoreo. Practicar deporte con mascarilla es posible, pero la respira- 
ción se podría ir deteriorando durante un ejercicio físico prolongado; por tanto, sería útil una investigación protocolizada para evitar cualquier riesgo de contagio, que estudie la implicación del uso de diferentes tipos de mascarillas en la saturación de oxígeno, acidificación en la sangre y eficiencia respiratoria. Por otra parte, se plantean futuras investigaciones que empleen diferentes tipos de mascarillas a diferentes alturas como método de entrenamiento de los músculos respiratorios, y que sean validados mediante pruebas de esfuerzo y medición directa de gases. De esta manera se podrá conocer de manera más exacta una mejoría sobre el valor de $\mathrm{VO}_{2}$ max debido al mayor esfuerzo inspiratorio y espiratorio. Por último, resaltar que existen muy pocos estudios comparativos sobre dispositivos de protección respiratoria durante el ejercicio físico, sería interesante conocer las diferentes condiciones de hipoxia e hipercapnia analizadas a través de la graduación de filtros o de las particularidades de cada mascarilla mientras se realiza ejercicio físico durante un tiempo prolongado.

\section{BIB LIOGRAFÍA}

Bellovary, B.N., King, K.E., Nunez, T.P., Mccormick, J.J., Wells, A.D., Bourbeau, K.C., Fennel, Z.J., Li, Z., Johnson, K.E., Moriarty, T., \& Mermier, C.M. (2019). Effects of high-intensity interval training while using a breathing-restrictive mask compared to intermittent hypobaric hypoxia. Journal of Human Sport and Exercise, 14(4), 821-833. doi:10.14198/jhse.2019.144.11

Birtwhistle, R., Pottie, K., Shaw, E., Dickinson, J.A., Brauer, P., Fortin, M., Bell, N., Singh, H., Tonelli, M., Connor,S., Lewin, G., Joffres, M., \& Parkin, P. (2012). Canadian Task Force on Preventive Health Care: we're back! Canadian Family Physician, 58, 13-5.

Bourouiba, L. (2020). Turbulent Gas Clouds and Respiratory Pathogen Emissions Potential Implications for Reducing Transmission of Covid-19. JAMA The Journal of the American Medical Association, 323(18), 1837-1838. doi:10.1001/jama.2020.4756

Chen, Y., Ebenstein, A.M., \& Greenstone, H.L. (2013). Evidence on the impact of sustained exposure to air pollution on life expectancy from China's Huai River policy, Proceedings of the National Academy of Sciences of the United States of America 110, 12936-12941.

Ciapponi, A. (2015). QUADAS-2: instrumento para la evaluación de la calidad de estudios de precisión diagnóstica. Revista Evidencia Actualización en la Práctica Ambulatoria, 18(1), 22-26.

Del Río Haza, F., López, O.G., Tobón, S.H., Leyva, P.D., \& García, R.S. (2020). Los aerosoles y los virus. II. La física del contagio aéreo de la Covid-19. Departamento de Física, Universidad Autónoma Metropolitana, Iztapalapa.

Freemas, J.A., Wilhite, D.P, Greenshields, J.T., Adamic, E.M., \& Mickleborough, T.D. (2020) Comparison between a facemask and mouthpiece on breathing mechanics and gas exchange variables during highintensity exercise. European Journal of Sport Science, 20(2), 211-218, doi:10.1080/17461391.2019.1628309

Guo, M., \& Fu, S. (2019). Running with a Mask? The Effect of Air Pollution on Marathon Runners' Performance. Journal of Sports Economics, 20(7), 903-928. doi:10.1177/1527002518822701

Hutton, B., Salanti, G., Caldwell, D. M., Chaimani, A., Schmid, C. H., Cameron, C., Loannidis, J. P. A., Straus, S., Thorlund, K., Jansen, J. P., Mulrow, C., Catalá-López, F., Gøtzsche, P. C., Dickersin, K., Boutron, I., Altman, D. A., \& Moher, D. (2015). The PRISMA extension statement for reporting of systematic reviews incorporating network meta-analyses of health care interventions: Checklist and explanations PRISMA extension for network meta-analysis. Annals of Internal Medicine, 162(11), 777-784. doi:10.7326/M14-2385

Jung, H.C., Lee, N.H., John, S.D., \& Lee, S. (2019). The elevation training mask induces modest hypoxaemia but does not affect heart rate variability during cycling in healthy adults. Biology of Sport, 36(2), 105-112. doi:10.5114/biolsport.2019.79976

Kao, T. W., Huang, K. C., Huang, Y. L., Tsai, T. J., Hsieh, B. S., \& Wu, M. S. (2004). The physiological impact of wearing an N95 mask during hemodialysis as a precaution against SARS in patients with end-stage renal disease. Journal of the Formosan Medical Association, 103(8), 624-628.

Leung, N., Chu, D., Shiu, E., Chan, K. H., McDevitt, J. J., Hau, B., Yen, H. L., Li, Y., Ip, D., Peiris, J., Seto, W. H., Leung, G. M., Milton, D. K., \& Cowling, B. J. (2020). Respiratory virus shedding in exhaled breath and efficacy of face masks. Nature medicine, 26(5), 676-680. doi:10.1038/s41591-020-0843-2

National Institute of Occupational Safety and Health (NIOSH). (2020). Approval Support, Test Procedures, Standards, and User Notices. Department of Health \& Human Services, U.S.A. Recuperado de https:// www.cdc.gov/niosh/npptl/respmanuf.html

Organización Mundial de la Salud. (2020). Advice on the use of masks in the context of Covid-19: interim guidance, 5 June 2020. World Health Organization. Recuperado de https://apps.who.int/iris/handle/ 10665/332293

Pifarré, F., Zabala, D.D., Grazioli, G., \& Maura, I. D. (2020). Covid-19 and mask in sports. Apunts Sports Medicine. doi:10.1016/j.apunsm. 2020.06.002

Porcari, J.P., Probst, L., Forrester, K., Doberstein, S., Foster, C., Cress, M.L., \& Schmidt, K. (2016). Effect of wearing the elevation training mask on aerobic capacity, lung function, and hematological variables. Journal of Sports Science and Medicine, 15(2), 379-386.

Pun, V. C., \& Manjouride, H.S. (2017). Association of ambient air pollution with depressive and anxiety symptoms in older adults: results from the NSHAP study. Environmental Health Perspectives 125, 342-348.

Rundell, K. W. (2012). Effect of air pollution on athlete health and performance. British Journal of Sports Medicine 46, 407-412.

Sellers, J.H., Monaghan, T.P., Schnaiter, J.A., Jacobson, B.H., \& Pope, Z.K. (2016). Efficacy of a Ventilatory Training Mask to Improve Anaerobic and Aerobic Capacity in Reserve Officers' Training Corps Cadets. Journal of Strength and Conditioning Research, 30(4), 11551160. doi:10.1519/JSC.0000000000001184

Tobin, B., Costalat, G., \& Renshaw, G.M.C. (2020). Intermittent not continuous hypoxia provoked haematological adaptations in healthy seniors: hypoxic pattern may hold the key. European Journal of Applied Physiology, 120, 707-718. doi:10.1007/s00421-020-04310-y

Toresdahl B.G. \& Asif, I.M. (2020). Coronavirus Disease 2019 (Covid-19): Considerations for the Competitive Athlete. Sports Health, 12(3), 221-224. doi:10.1177 / 1941738120918876

Wagner, D.R., \& Clark, N.W. (2016). Similar results for face mask versus mouthpiece during incremental exercise to exhaustion. Journal of Sports Sciences, 34(9), 852-855.

Whittemore, R, Chao, A, Jang, M., Minges, K.E., \& Park, C. (2014). Methods for knowledge synthesis: an overview. Heart Lung,43(5), 453-61. doi:10.1016/j.hrtlng.2014.05.014

Wong, A. Y., Ling, S. K., Louie, L. H., Law, G. Y., So, R.C., Lee, D.C., Yau, F.C., \& Yung, P.S. (2020). Impact of the Covid-19 pandemic on sports and exercise. Asia-Pacific Journal of Sports Medicine, Arthroscopy, Rehabilitation and Technology, 22, 39-44. doi:10.1016/j.asmart.2020.07.006

Zhou, B., Costa, A. B., \& Lukowicz, P. (2020). Accurate spirometry with integrated barometric sensors in face-worn garments. Sensors (Switzerland), 20(15), 1-22. doi:10.3390/s20154234

Zhu, N., Zhang, D., Wang, W., Li, X., Yang, B., Song, J., Zhao, X., Huang, B., Shi, W., Lu, R., Niu, P., Zhan, F., Ma, X., Wang, D., Xu, W., Wu, G., Gao, G. F., Tan, W., \& China Novel Coronavirus Investigating and Research Team (2020). A Novel Coronavirus from Patients with Pneumonia in China, 2019. The New England journal of medicine, 382(8), 727-733. doi:10.1056/NEJMoa2001017 


\section{The use of masks in sport: an integrative review during Covid-19}

\section{El uso de mascarilla en el deporte: una revisión integradora durante la Covid-19 Joel Manuel Prieto Andreu}

Universidad Internacional de la Rioja. España.

CORRESPONDENCIA:

Joel Manuel Prieto Andreu

joelmanuel.prieto@unir.net

Recepción: septiembre 2020 • Aceptación: enero 2021

\section{CÓMO CITAR EL ARTÍCULO:}

Prieto, J.M. (2021). The use of masks in sport: an integrative review during Covid-19. Cultura, Ciencia y Deporte, 16(49), $393-$ 410. http://dx.doi.org/10.12800/ccd.v16i49.1554

\begin{abstract}
The use of the mask in the sports field has been studied to improve the athlete's performance and its protection against a contaminated environment, such as the one existing in the current pandemic panorama of Covid-19. This review analyzes the impact of the mask on different physiological factors while doing physical exercise. A random selection was made of 2,420 studies published in Scholar Google, 118 in SCOPUS and 137 studies published in WOS between the periods 2016-2020, with $95 \%$ confidence and +/- 4.3 sampling error, which served for the selection and final analysis of 10 studies chosen intentionally following CTFPHC levels of evidence and the methodological rigor of a structured classification system called PEDro Scale. The results highlight that the use of masks in athletes causes hypoxic and hypercapnic breathing at a certain intensity; the use of masks such as the Elevation Training Mask (ETM) trains the respiratory muscles but does not improve lactic anaerobic performance as intermittent hypoxic training (IHT) does in height. It is discussed about hypoxia and hypercapnia conditions and the use of masks to improve physical performance in athletes.
\end{abstract}

Kew words: mask, sport, hypoxia, hipercapnia, Covid-19.

\section{Resumen}

El uso de la mascarilla en el ámbito deportivo se ha estudiado para mejorar el rendimiento del deportista y como protección ante un ambiente contaminado, como el existente en el panorama pandémico actual de la Covid-19. La presente revisión analiza la repercusión de la mascarilla en diferentes factores fisiológicos mientras se hace ejercicio físico. Se hizo una selección aleatoria de 2.420 estudios publicados en Google Académico, 118 en SCOPUS y de 137 publicados en WOS entre los períodos de 2016-2020, con el $95 \%$ de confianza y +/- 4,3 de error muestral, el cual sirvió para la selección y análisis final de 10 estudios escogidos intencionalmente siguiendo los niveles de evidencia del CTFPHC y el rigor metodológico de un sistema de clasificación estructurado llamado PEDro Scale. Los resultados destacan que el uso de mascarillas en deportistas provoca respiración hipóxica e hipercápnica a una intensidad determinada; el uso de mascarillas como la Elevation Training Mask (ETM) entrena los músculos respiratorios, pero no mejora el rendimiento anaeróbico láctico, como sí lo hace el entrenamiento hipóxico intermitente (IHT) en altura. Se discute sobre las condiciones de hipoxia e hipercapnia y el uso de las mascarillas para la mejora del rendimiento físico en deportistas.

Palabras clave: mascarilla, deporte, hipoxia, hipercapnia, Covid-19. 


\section{Introduction}

Research on the use of masks in sports can be specified in two aspects: studies that use masks to simulate a certain altitude to improve the athlete's performance (Bellovary et al., 2019; Porcari, et al., 2016; Sellers, Monaghan, Schnaiter, Jacobson \& Pope, 2016); and recent studies that use them as a protection factor against a contaminated environment or that examine their physiological repercussions in a single group or by comparing a group with a certain type of mask with a control group (Freemas, Wilhite, Greenshields, Adamic \& Mickleborough, 2020 ; Guo, \& Fu, 2019; Jung, Lee, John \& Lee, 2019; Pifarré, Zabala, Grazioli \& Maura, 2020; Tobin, Costalat \& Renshaw, 2020; Wagner \& Clark, 2016; Wong, 2020). In the pandemic panorama of year 2020, in which the obligation to wear a mask has been established, this review aims to analyze the literature on the use of the mask, considering its repercussions while doing physical exercise.

There are studies looking at the effects in athletes of wearing specialized masks that restrict airflow and simulate training at altitude. Altitude training and Respiratory Muscle Training (RMT) can improve performance in elite athletes, various devices have been developed to help athletes gain an advantage in competition, such as the Elevation Training Mask 2.0 (ETM). ETM supposedly simulates training at altitude and has been suggested to increase aerobic capacity (VO2max), endurance performance, and lung function. According to the results of Porcari et al. (2016) use of the ETM may improve specific markers of endurance performance, Maximal Oxygen Consumption (VO2max), Ventilatory Threshold (VT), and Respiratory Compensation Threshold (RCT), beyond the improvements seen with interval training alone. However, following Jung, Lee, John and Lee (2019), future studies are needed to determine whether repeated exposure to high intensity exercise with an ETM provides benefits similar to those of altitude training.

Regarding the type of masks, the resistance to breathing can be determined by the thickness of the material, the size of the mask, the intensity of the exercise, the open or closed environment, and the total or partial coverage of the nose and mouth. On the other hand, the use of masks to protect against microbial agents, whether surgical, hygienic or with a FFP1-3 particle filter (they filter between $78 \%$ and $98 \%$ of the particles), the use of integral respirators with P100 filters (up to $99.2 \%$ protection of mucous membranes and viral joints), or the N95 that retain $95 \%$ of the 0.3 $\mu \mathrm{m}$ (micrometer) particles, being those recommended by the National Institute of Occupational Safety and Health (NIOSH) (2020), can be affected by humidity, since during exercise a warm and humid microclimate is created in which nasal secretions also proliferate, being more recommended than surgical or specialized masks the use of synthetic fabrics that reduce heat, even if they present a lower barrier to the entry and exit of germs. On the other hand, in a climate polluted by aerosols or droplets with a viral load spread in the form of a cloud, the use of masks would be inefficient, since the ocular mucosa would not be protected with this type of virulent dispersion. Based on turbulence studies (Bourouiba, 2020), the integrity and isolation capacity of ocular lenses as protection mechanisms against viral dispersions have yet to be evaluated.

At present, after just over half a year since the state of alarm began in Spain, there is uncertainty about how long SARS-COV-2 (Covid-19 disease) remains in the air when sneezing or when they spread in the air aerosol virus particles. Following the research of Leung et al. (2020) surgical masks can effectively reduce the emission of influenza virus particles to the environment in respiratory droplets, but not in aerosols. According to NIOSH (2020) the use of masks or respirators will depend on the protection capacity that is needed against an environment contaminated with a certain viral load.

The elasticity of the mask itself can also reduce its antimicrobial capacity. A 3-layer surgical mask, of which the central one is a "Melt Brown" layer, has a porosity that prevents the passage of $0.1 \mu \mathrm{m}$ microparticles, an FFP2 or N95 of $0.3 \mu \mathrm{m}$ and an FFP3 of $0.023 \mu \mathrm{m}$ (Zhu et al., 2020), for example, the size of SARS-CoV-2 is between 0.06 and $0.14 \mu \mathrm{m}$, on the other hand carbon dioxide (CO2) is $0.000232 \mu \mathrm{m}$. Therefore, SARS$\mathrm{CoV}-2$ is approximately 500 times larger than a CO2 molecule. However, if the mask wrinkles or stretches during exercise, this porosity may be affected, so that its antimicrobial protective capacity could be impaired during exercise. On the other hand, the behavior of particles with a size smaller than $0.3 \mu \mathrm{m}$ is less predictable than that of large particles, the behavior of particles smaller than $0.3 \mu \mathrm{m}$ can be described through Brownian motion, a phenomenon whereby particles with this mass are small enough to be unimpeded when traveling in the air, describing an erratic pattern when they interact with other molecules in the air such as nitrogen or oxygen, being more difficult to capture by the filters of masks (Del Río Haza, López, Tobón, Leyva, \& García (2020). Following Guo, and Fu (2019), suspended particles of less than $2.5 \mu \mathrm{m}$ (PM2.5), such as SARSCoV-2 measuring between 0.08 and $0.2 \mu \mathrm{m}$, seem to be a better indicator of urban pollution than those that 
Table 1. Randomized and stratified selection of records in WOS and SCOPUS.

\begin{tabular}{|c|c|c|c|c|c|c|}
\hline \multirow[t]{2}{*}{ Year } & \multicolumn{2}{|c|}{ Articles } & \multicolumn{2}{|c|}{ Proportion } & \multicolumn{2}{|c|}{ Stratum sample } \\
\hline & wos & SCOPUS & wos & SCOPUS & wos & SCOPUS \\
\hline 2020 & 13 & 19 & $9,6 \%$ & $16,1 \%$ & 4 & 5 \\
\hline 2019 & 39 & 29 & $28,5 \%$ & $24,6 \%$ & 8 & 7 \\
\hline 2018 & 31 & 24 & $22,6 \%$ & $20,3 \%$ & 10 & 4 \\
\hline 2017 & 30 & 23 & $21,8 \%$ & $19,5 \%$ & 7 & 8 \\
\hline 2016 & 24 & 23 & $17,5 \%$ & $19,5 \%$ & 5 & 6 \\
\hline
\end{tabular}

Source: Prepared by the author, from data extracted in WoS and SCOPUS.

have been used up to now, PM10 (particulate matter of $10 \mu \mathrm{m}$ or less in diameter), this is because their origin is anthropogenic in a high proportion, that is the reason what pollutants are the result of human activities.

According to Guo, and $\mathrm{Fu}$ (2019), long-term exposure to particulate matter $2.5 \mu \mathrm{m}$ or less in diameter (PM2.5), less than $10 \mu \mathrm{m}$ (PM10), sulfur dioxide (SO2), nitrogen dioxide (NO2), carbon monoxide (CO) and ozone (O3), can cause cardiopulmonary diseases. Following Chen, et al. (2013), such exposure could cause respiratory infections, lung cancer, decreased lung function, irregular heartbeats, increased respiratory problems, non-fatal heart attacks, angina, childhood morbidity, asthma, and hope of reduced life. Along the same lines, air pollution can also reduce cognitive ability, increase anxiety and have other negative psychological effects (Pun, \& Manjouride, 2017). Additionally, the sports health literature also provides evidence on the negative effect of air pollution on the health and performance of athletes (Rundell, 2012).

The present review analyzes the existing literature on the use of the mask in sports in the main JCR and SCOPUS databases. The following questions are posed: Does the use of masks during physical exercise affect performance? Is it safe for athletes to exercise with a mask? The 10 articles analyzed in the results of the review study the use of the mask and its impact on different physiological factors while doing physical exercise. Hypoxia and hypercapnia conditions and the use of masks to improve physical performance in athletes are discussed.

\section{Method}

The review is integrative with a systematic approach focused on the methodological movement of EvidenceBased Practice (EBP) (Whittemore, Chao, Jang, Minges, \& Park, 2014). It has a quali-quantitative character, whose main data collection technique was the content analysis of each of the randomly selected records, which were part of the stratified sample applied for this purpose. This type of integrative review is mainly focused on synthesizing theoretical knowledge and scientific evidence on the repercussions of the use of the mask in the sports field. This review followed the guidelines of the PRISMA statement for systematic reviews in order to structure the correct development of the manuscript (Hutton et al. 2015).

The study population focused on all those Open Access publications, registered and identified in ISI Web of Science (WoS) and SCOPUS, with the search terms in their titles, abstracts or keywords: mask and sport. These words were combined as follows: mask AND sport. The use of these terms in English was made in view that it is the language in which all the works indexed in WoS have records. This reduces inadvertent skipping of sensitive scan cases. On the other hand, the search was limited to the last 5 years (from January 2016 to August 2020) because it was considered a recent scientific contribution and due to the excess of references in the last 10 years on literature that used the use of masks to improve the performance of the athlete, as well as the documentary silence in recent years on the use of masks as protection against a contaminated environment, literature that has proliferated in 2020 due to the pandemic.

The centrality of the study around WoS was motivated by the fact that it is one of the databases with the largest number of journals and scientific recognition today, which makes it the most exhaustive in the world; in addition to being the oldest and with the highest academic impact internationally recognized, being 24,891 registered journals if the Core reviews of Web of Science are taken into account, as opposed to Scopus, which follows with 24,701 registered journals. In total, 137 records were identified in WoS and 118 in SCOPUS. From this total, a random selection of 34 records was made in WOS and 30 in SCOPUS, distributed in a stratified manner, with $95 \%$ confidence and $+/-4.3$ sampling error (Table I). This selection was made starting from a table of random numbers created adhoc by the author, which served to select each case study by year, assigning them a unique identification number (see Table 1). 


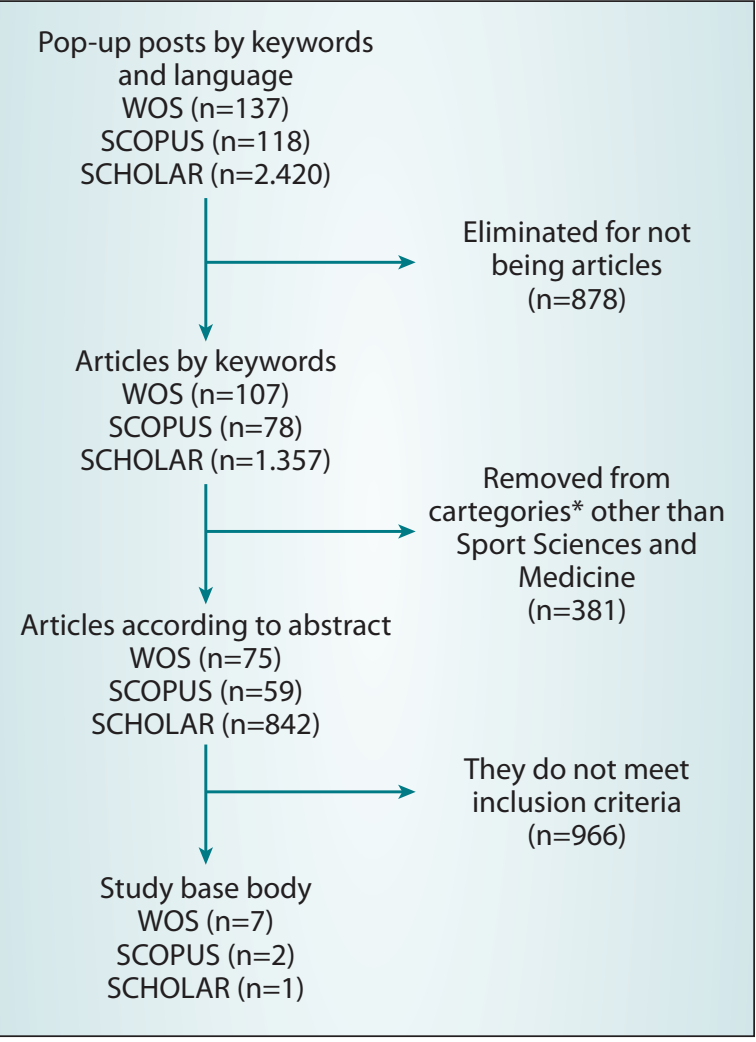

Figure 1. Flowchart of the selection of the study base body.

Another search was carried out from January 2016 to August 2020 in Scholar Google, a search engine specialized in the search for scientific-academic content and bibliography. The keywords used for the Scholar Google search were: mask AND sport. Of the subtotal of records in Scholar without including citations or patents $(2,420)$, WOS (137) and SCOPUS (118), 10 studies were included in the integrative review in the last phase of article inclusion, as can be seen in the fluxogram of figure 1.

The PICR reference (Participants / Intervention / Comparison / Results) and the following inclusion criteria were applied: peer-reviwed and Open Access studies with full-text access, experimental, randomized and controlled with experimental group (with mask) and control (without mask); The different physiological parameters of measurement are reflected and the results have been obtained with scales and measurement units that have been provided by the studies; published works from 2015 onwards, in all geographic contexts, and written in Spanish and English; articles belonging eminently to the categories* of Sport sciences, and to a lesser extent to the Pharmacology Pharmacy, Chemistry Analytical, Biochemical Research Methods and Enginnering Electrical Electronic categories. The participants in the studies analyzed in the review were athletes (moderately trained men and women).
Regarding the intervention criteria, experimental studies were selected and epidemiological studies and reviews were discarded. Regarding the comparison criterion, the studies that included those that determined two groups were taken into account, the group of athletes who wore a mask and went through some training process or stress test, and the control group that did not experience it. Finally, under the results criterion, those studies that statistically demonstrated the influence of the use or not of the mask and its repercussions on different physiological parameters were taken into account.

Regarding the exclusion criteria, conference communications published in the minutes book, works from unreliable academic sources, references prior to 2015, works belonging to institutional repositories and publications in popular journals, reviews, theses or presentations have been omitted. On the other hand, the methodological rigor was evaluated using a structured classification system called the PEDro Scale (Verhagen et al. 1998) used to quantitatively assess the external validity, the methodological quality and the statistical description of experimental randomized studies.

On the other hand, the levels of evidence, internal validity and degrees of recommendation were evaluated through the Canadian Task Force on Preventive Health Care (CTFPHC) (2012). Lastly, the probability of biases and concern about the applicability of the results was evaluated with the Quadas-2 instrument (Ciapponi, 2015).

\section{Results}

Of the 10 articles analyzed, 7 of them were published in JCR journals. The $\mathrm{H}$ index and the quartile of each journal was obtained through the 2019 Scimago Ranking in the Sports Science category. $40 \%$ of the articles belong to journals located in the Q1 quartile, $30 \%$ in Q3, 20\% in Q2 and 10\% in Q4. Following the QUADAS-2 instrument for assessing the quality of diagnostic precision studies, all the studies analyzed in the review have a low probability of bias and raise little concern regarding their applicability (see Figure 2).

Regarding the PEDro Scale, the PEDro scale of 11 criteria quickly identifies which of the randomized trials may have sufficient internal validity (criteria 2-9), external validity (criterion 1 : information specified or not on the choice of subjects) and sufficient statistical information to make the results interpretable (criteria 10 and 11). $40 \%$ of the articles meet 5 or fewer criteria, $40 \%$ meet between 7 or 8 criteria and $20 \% 9$ or more criteria. 


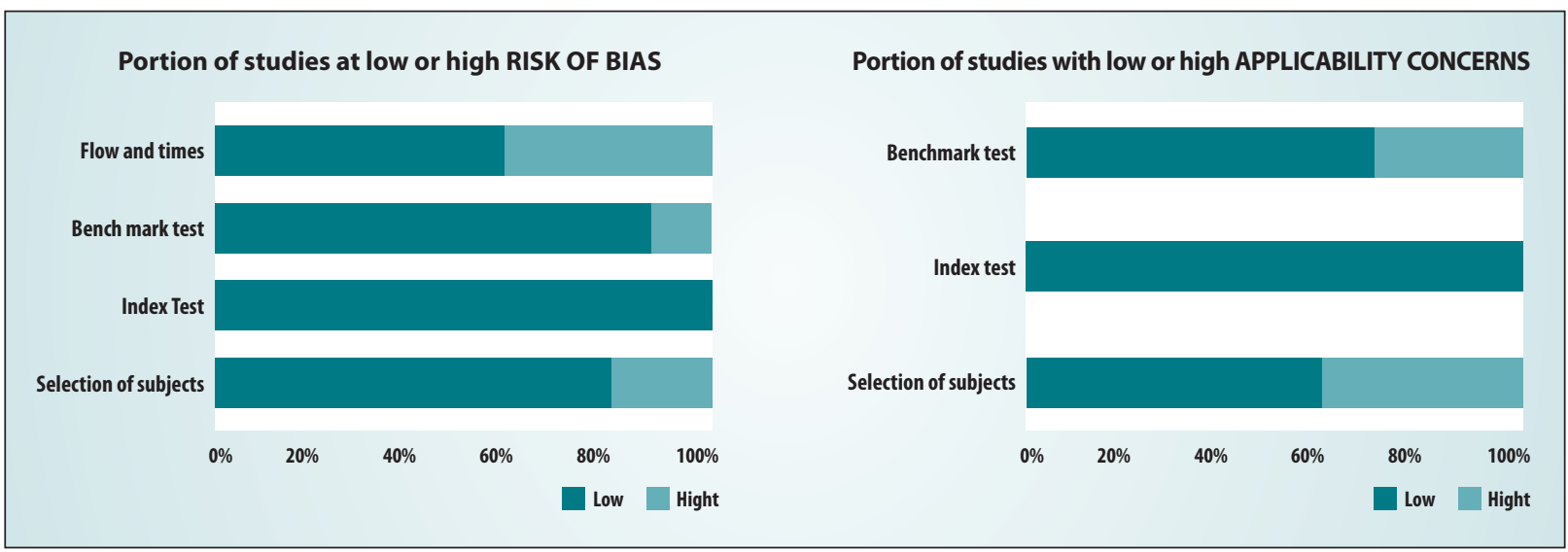

Figure 2. Studies with risk of bias and concerns about low or high applicability.

In all studies informed consent was applied to all participants. $80 \%$ of the studies have internal and external validity, have good evidence for recommendation and the results are easily interpretable in most of them. However, most of the studies with a single group, as well as the studies that compared the control group (without mask) with the experimental group (with mask), did not blind either the participants or the evaluators, in addition, the masking effect it did not take place in most of the studies analyzed since the group assignment was not hidden, so internal validity was reduced in this regard and the number of criteria met in the PEDro Scale was reduced. The data extracted from the synthesis were inserted in Table 2.

\section{Discussion}

The literature that has studied the conditions of hypoxia and hypercapnia using a mask during physical exercise is limited and contemporary (Jung, 2019; Pifarré, 2020; Tobin, Costalat \& Renshaw, 2020; Wong, 2020). Following Jung et al. (2019) observed in the mask trial a greater response in resting systolic blood pressure and a higher heart rate, while pulse oximetry saturation was lower during the cycling test with an intensity of $70 \%$ of $\mathrm{VO} 2$ peak in compared with the trial without a mask, concluding that high intensity cycling induces moderate hypoxemia, delaying the autonomic cardiac recovery from exercise. Along the same lines, in the study by Pifarré et al. (2020) where they evaluated the physiological impact of hypercapnia and hypoxia generated by masks during aerobic sports, they recorded a decrease in $\mathrm{O} 2$ (hypoxia) and an increase in CO2 (hypercapnia) before, during and after exercise, highlighting a decrease in post-exercise arterial $\mathrm{O} 2$ saturation. The authors concluded that the use of masks during a brief exercise with an intensity of around
6-8 METS, decreases $\mathrm{O} 2$ by $3.7 \%$ and increases $\mathrm{CO} 2$ concentration, although it was not an ergo-spirometric analysis, but an analysis on the composition soft drink inhaled by the study population during exercise with a mask. Probably, during moderate-high physical exercise the speed of the inspired and exhaled air against the mask is higher, so the mask could widen your pores. On the other hand, the exhaled $\mathrm{CO} 2$ molecule, being so small $(0.000232 \mu \mathrm{m})$, can pass through any surgical mask or mask with a particle filter. However, during physical exercise there is a higher respiratory rate and the renewed air in each breath per unit of time is lower compared to a basal state. Probably, with the mask, the respiratory cycle tends to be longer and the subjective perceived effort could be greater. Along the same lines, in the study by Wong et al. (2020) concluded that wearing a mask significantly increases heart rate and perceived exertion just walking at $4 \mathrm{~km} / \mathrm{h}$.

Regarding the use of masks to improve performance in athletes, in this review it was considered whether the use of masks during physical exercise affects performance. Following Porcari et al. (2016) only the group that used the Elevation Training Mask (ETM) had significant improvements in the ventilatory threshold, power production and in the respiratory compensation threshold, concluding that the use of the ETM while participating in a cycle ergometer training program the 6-week high intensity does not appear to act as an altitude simulator, but more as a respiratory muscle training device. On the other hand, the study by Sellers et al. (2016) found that, between the groups with and without a mask, there were no differences in the fatigue index, anaerobic capacity, maximum power, and Vo2 max. or in the time to exhaustion, results that confirmed that the use of the ventilatory training mask during training did not provoke superior aerobic or anaerobic adaptations in the cadets of the Reserve Officers Training Corps (CEOR). Sellers et al. (2016) 
Table 2. Synthesis of results of the integrative review.

\begin{tabular}{lllll}
\hline Study & Methodological Rigor & Objective & Participants & Proce \\
\hline 1-Wagner, D.R., \& & - PEDro Scale: 7 & Compare & 14 volunteers, 7 & They \\
Clark, N.W. (2016). & - Recomendation: A & metabolic gas & men and 7 women & ran \\
Similar results & - Level of evidence: I & analysis data & (25.1 2.7 years). & tre \\
for face mask & - Internal validity: B & between nose clip & & w \\
versus mouthpiece & - External validity: si & mouthpiece and & & and \\
during incremental & - Quotes in scholar: 7 & mask. & & \\
exercise to & - Magazine: Journal of & & & \\
exhaustion. & Sports Sciences & & & \\
& - H index: 128 (Q1) & & & \\
& & & &
\end{tabular}

\begin{tabular}{ll} 
Process & Measurement parameters \\
\hline They completed two & -Maximal Oxygen Consumption \\
random maximal & (VO2max) \\
treadmill tests in 1 & - Minute ventilation (VE) \\
week, a nasal clip test & - Fraction of expired oxygen (FEO2) \\
and a mask test. & - Carbon Dioxide (FECO2) \\
& - Respiration Rate (RR) \\
& - Tidal Volume (Vt) \\
& - Heart Rate (HR) \\
& - Rating of Perceived Exertion (RPE)
\end{tabular}

Results and conclusions

The difference in maximum oxygen consumption (V02max) between the nasal clip $(52.7 \pm 11.3 \mathrm{ml} \cdot \mathrm{kg}$ $-1 . \mathrm{min}-1)$ and the mask $(52.2 \pm$ $11.7 \mathrm{ml} \cdot \mathrm{kg}-1 \cdot \min -1$ ) was not significant $(p=.53)$. Furthermore, the differences between nasal clip and mask in the rest of the parameters - Rating of Perceived Exertion (RPE) were not significant $(p>.05)$. The selection of nasal clip or mask does not affect gas exchange or the participant's breathing patterns.

\begin{tabular}{lll}
\hline 2- Porcari, J.P. et & -PEDro Scale: 10 & Know the effects \\
al. (2016). Effect & - ORecomendation: A & of the Elevation \\
of wearing the & - Level of evidence: I & Training Mask \\
elevation training & - Internal validity: B & 2.0 (ETM), which \\
mask on aerobic & - External validity: $\mathrm{si}$ & supposedly \\
capacity, lung & - Quotes in scholar: 51 & simulates training \\
function, and & - Magazine: Journal of & at altitude. \\
hematological. & Sports Science and & \\
variables & Medicine & \\
& - H index: 58 (Q2) &
\end{tabular}

$\begin{array}{lll}25 \text { volunteers (16 } & \text { They completed } & \text {-Maximal Oxygen Consumption } \\ \text { men and 9 women) } & 6 \text { weeks of high } & \text { (VO2max) } \\ \text { moderately trained. } & \text { intensity cycle } & \text { - Ventilatory Threshold (VT) } \\ & \text { ergometer training, } & \text { - Respiratory Compensation } \\ & \text { the control group } & \text { Threshold (RCT) } \\ & \text { without a mask (12) } & \text { - Pulmonary function } \\ & \text { and the experimental } & \text { - Maximal inspiration pressure } \\ & \text { group with a } & \text { - Hemoglobin } \\ & \text { mask (12) were } & \text { - Hematocrit } \\ & \text { randomized. } & \end{array}$

There was a significant improvement in V02max in both the control group $(13.5 \%)$ and the mask group $(16.5 \%)$. Only the mask group had significant improvements in

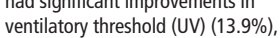
power output (PO) in UV (19.3\%), respiratory compensation threshold respiratory compensation threshold
(UCR) $(10.2 \%)$ and PO in the UCR (16.4\%) before and after the test. Using the ETM does not appear to act as an altitude simulator, but more as a respiratory muscle training device.

\begin{tabular}{|c|c|c|c|c|c|c|}
\hline $\begin{array}{l}\text { 3- Sellers, J.H.,, } \\
\text { Monaghan, T.P., } \\
\text { Schnaiter, J.A.,' } \\
\text { Jacobson, B.H., \& } \\
\text { Pope, Z.K. (2016). } \\
\text { Efficacy of a } \\
\text { Ventilatory Training } \\
\text { Mask to Improve } \\
\text { Anaerobic and } \\
\text { Aerobic Capacity } \\
\text { in Reserve Officers' } \\
\text { Training Corps } \\
\text { Cadets. }\end{array}$ & $\begin{array}{l}\text { - PEDro Scale: } 8 \\
\text { - oRecomendation: A } \\
\text { - Level of evidence: I } \\
\text { - Internal validity: B } \\
\text { - External validity: si } \\
\text { - Quotes in scholar: } 11 \\
\text { - Magazine: Journal } \\
\text { of Strength and } \\
\text { Conditioning Research } \\
\text { - H index: } 121 \text { (Q1) }\end{array}$ & $\begin{array}{l}\text { To examine } \\
\text { the efficacy of } \\
\text { a ventilatory } \\
\text { training mask } \\
\text { for improving } \\
\text { anaerobic / } \\
\text { aerobic fitness in } \\
\text { cadets. }\end{array}$ & $\begin{array}{l}17 \text { cadets from } \\
\text { the Reserve } \\
\text { officer Training } \\
\text { Corps (CEOR) of } \\
\text { a Midwestern } \\
\text { university. }\end{array}$ & $\begin{array}{l}\text { Physical training for } \\
6 \text { weeks, the control } \\
\text { group without a } \\
\text { mask (8) and the } \\
\text { experimental group } \\
\text { with a mask (9) } \\
\text { with adjustment to } \\
\text { simulate an altitude } \\
\text { of } 2750 \mathrm{~m} \text {. }\end{array}$ & $\begin{array}{l}\text {-Maximal Oxygen Consumption } \\
\text { (VO2max) } \\
\text { - Anthropometry } \\
\text { - 30" Wingate Anaerobic Test } \\
\text { (WAnT) } \\
\text { - Fatigue index }\end{array}$ & $\begin{array}{l}\text { There was no significant effect ( } \mathrm{p} \\
\leq 0.05 \text { ) between groups on fatigue } \\
\text { index, anaerobic capacity, maximal } \\
\text { power, Vo } 2 \text { max. or in time to } \\
\text { exhaustion. These results suggest } \\
\text { that the use of the ventilatory } \\
\text { training mask during training did not } \\
\text { elicit superior aerobic or anaerobic } \\
\text { adaptations in CEOR cadets. }\end{array}$ \\
\hline $\begin{array}{l}\text { 4- Guo, M., \& Fu, } \\
\text { S. (2019). Running } \\
\text { With a Mask? } \\
\text { The Effect of } \\
\text { Air Pollution on } \\
\text { Marathon Runners' } \\
\text { Performance }\end{array}$ & $\begin{array}{l}\text { - PEDro Scale: } 4 \\
\text { - 'Recomendation: M } \\
\text { - Level of evidence: II-2 } \\
\text { - Internal validity: M } \\
\text { - External validity: si } \\
\text { - Quotes in scholar: } 7 \\
\text { - Magazine: Journal of } \\
\text { Sports Economics } \\
\text { - H index: } 44 \text { (Q3) }\end{array}$ & $\begin{array}{l}\text { Relate the level of } \\
\text { air pollution with } \\
\text { the performance } \\
\text { of the runners } \\
\text { through a } \\
\text { mathematical } \\
\text { model. }\end{array}$ & $\begin{array}{l}314,341 \text { popular } \\
\text { marathon runners } \\
\text { in } 37 \text { countries in } \\
56 \text { running events } \\
\text { in China between } \\
2014 \text { and } 2015 .\end{array}$ & $\begin{array}{l}\text { Data from the Daily } \\
\text { Air Quality Index } \\
\text { (IDCA) comes from } \\
\text { China's Ministry } \\
\text { of Environmental } \\
\text { Protection and } \\
\text { meteorological data } \\
\text { from the Bloomberg } \\
\text { database. }\end{array}$ & $\begin{array}{l}\text { - Index of } 6 \text { pollutants: particulate } \\
\text { matter less than } 2.5 \text { microns } \\
\text { (PM2.5) and less than } 10 \text { microns } \\
\text { (PM10), sulfur dioxide (SO2), } \\
\text { nitrogen dioxide (NO2), carbon } \\
\text { monoxide (CO ) and ozone (03). } \\
\text { - Mathematical formula: pollution } \\
\text { index, weather conditions, } \\
\text { temperature, wind speed, relative } \\
\text { humidity and precipitation, } \\
\text { filtering by age and gender. }\end{array}$ & $\begin{array}{l}\text { The standard established by } \\
\text { the World Health Organization } \\
\text { (WHO) for PM2.5, PM10 and SO2 } \\
\text { concentrations is } 25,50 \text { and } 20 \text { ug } \\
\text { / } \mathrm{m} 3 \text { on average every } 24 \text { hours, } \\
\text { respectively. However, the means } \\
\text { during running hours in the study } \\
\text { sample were approximately } 74, \\
105, \text { and } 22 \text { ug / } 33 \text {, suggesting } \\
\text { deleterious effects on the } \\
\text { performance of the runners. }\end{array}$ \\
\hline $\begin{array}{l}\text { 5- Jung, H.C., Lee, } \\
\text { N.H., John, S.D., \& } \\
\text { Lee, S. (2019). The } \\
\text { elevation training } \\
\text { mask induces } \\
\text { modest hypoxaemia } \\
\text { but does not affect } \\
\text { heart rate variability } \\
\text { during cycling in } \\
\text { healthy adults }\end{array}$ & $\begin{array}{l}\text { - PEDro Scale: } 8 \\
\text { - oRecomendation: A } \\
\text { - Level of evidence : I } \\
\text { - Internal validity: B } \\
\text { - External validity: si } \\
\text { - Quotes in scholar: } 3 \\
\text { - Magazine: Biology } \\
\text { of Sport } \\
\text { - H index: } 23 \text { (Q2) }\end{array}$ & $\begin{array}{l}\text { To examine } \\
\text { the effects of } \\
\text { elevation training } \\
\text { mask (ETM) on } \\
\text { hemodynamics } \\
\text { and HR in healthy } \\
\text { cyclists. }\end{array}$ & $\begin{array}{l}20 \text { initial volunteers, } \\
\text { but including } 15 \\
\text { healthy cyclists (9 } \\
\text { men and } 6 \text { women } \\
\text { aged } 27.0 \pm 1.14 \\
\text { years) as selection } \\
\text { criteria: } \% \text { fat } \\
-30 \% \text { and without } \\
\text { pathologies. }\end{array}$ & $\begin{array}{l}\text { They completed } 2 \\
40 \text { "cycling training } \\
\text { sessions, with and } \\
\text { without a mask, } \\
\text { with } 3 \text { phases of } \\
10 \text { : } 50 \% \text { V02peak, } \\
70 \% \text { VO2peak and } \\
\text { recovery. An ECG } \\
\text { Actiwave-Cardio } \\
\text { monitor was used } \\
\text { to measure the } \\
\text { variables. }\end{array}$ & $\begin{array}{l}\text { - Blood pressure } \\
\text { - Heart Rate (HR) } \\
\text { - Pulse Oximetry Saturation (SPO2) }\end{array}$ & $\begin{array}{l}\text { A greater response was observed in } \\
\text { systolic blood pressure }(p=.035) \text { at } \\
\text { rest, a higher heart rate }(p=.047) \text {, } \\
\text { a shorter interval between beats } \\
\text { during recovery }(p=.01) \text { and a } \\
\text { lower SPO2 ( } p=.033) \text { during high } \\
\text { intensity cycling ( } 70 \% \text { of VO2peak) } \\
\text { in the mask test. The use of an ETM } \\
\text { during high intensity cycling (70\% } \\
\text { of V02peak) induces moderate } \\
\text { hypoxemia, delaying autonomous } \\
\text { cardiac recovery from exercise. }\end{array}$ \\
\hline $\begin{array}{l}\text { 6- Bellovary, B.N. et } \\
\text { al. (2019). Effects } \\
\text { of high-intensity } \\
\text { interval training } \\
\text { while using a } \\
\text { breathing-restrictive } \\
\text { mask compared } \\
\text { to intermittent } \\
\text { hypobaric hypoxia }\end{array}$ & $\begin{array}{l}\text {-PEDro Scale: } 8 \\
\text { - }{ }^{\circ} \text { Recomendation: A } \\
\text { - Level of evidence : I } \\
\text { - Internal validity: B } \\
\text { - External validity: sí } \\
\text { - Quotes in scholar: } 2 \\
\text { - Magazine: Journal } \\
\text { of Human Sport and } \\
\text { Exercise } \\
\text { - H index: } 23 \text { (Q3) }\end{array}$ & $\begin{array}{l}\text { To determine } \\
\text { the effects of } \\
\text { ETM compared } \\
\text { to intermittent } \\
\text { hypoxic training } \\
\text { (IHT) on aerobic } \\
\text { performance and } \\
\text { cycling economy. }\end{array}$ & $\begin{array}{l}30 \text { volunteer } \\
\text { cyclists without } \\
\text { hypertension } \\
\text { or previous } \\
\text { diseases (15 men } \\
\text { and } 15 \text { women) } \\
\text { randomized into } 3 \\
\text { groups: ETM (10), } \\
\text { IHT (10) and control } \\
\text { group (10). }\end{array}$ & $\begin{array}{l}\text { They performed two } \\
20 \text { 'HIIT tests / week } \\
\text { in } 6 \text { weeks measuring } \\
\text { V02max on a cycle } \\
\text { ergometer with active } \\
\text { recovery from } 90^{\prime} \text { 'to } \\
25 \mathrm{~W}(10 \text { episodes) } \\
\text { with } 5^{\prime} \text { input and } \\
\text { output. }\end{array}$ & $\begin{array}{l}\text { - Maximal Oxygen Consumption } \\
\text { (VO2max) } \\
\text { - Submaximal Power Output (PO) of } \\
100,125 \text { and } 150 \mathrm{~W}\end{array}$ & $\begin{array}{l}\text { All participants improved V02max } \\
\text { and PO at the ventilatory threshold } \\
\text { before and after training. The ETM } \\
\text { and IHT groups responded similarly } \\
\text { to maximal and submaximal effort } \\
\text { after six weeks of training. The } \\
\text { IHT group, but not the ETM group, } \\
\text { experienced an increase in glycolytic } \\
\text { energy during submaximal exercise. }\end{array}$ \\
\hline $\begin{array}{l}\text { 7- Freemas, J.A., } \\
\text { Wilhite, D.P, } \\
\text { Greenshields, J.T., } \\
\text { Adamic, E.M., \& } \\
\text { Mickleborough, T.D. } \\
\text { (2020). Comparison } \\
\text { between a } \\
\text { facemask and } \\
\text { mouthpiece } \\
\text { on breathing } \\
\text { mechanics and gas } \\
\text { exchange variables } \\
\text { during high- } \\
\text { intensity exercise }\end{array}$ & $\begin{array}{l}\text { - PEDro Scale: } 9 \\
\text { - }{ }^{\circ} \text { Recomendation: A } \\
\text { - Level of evidence : I } \\
\text { - Internal validity: B } \\
\text { - External validity: si } \\
\text { - Quotes in scholar: } 1 \\
\text { - Magazine: European } \\
\text { Journal of Sport } \\
\text { Science } \\
\text { - H index: } 47 \text { (Q1) }\end{array}$ & $\begin{array}{l}\text { To compare } \\
\text { respiratory } \\
\text { mechanics, } \\
\text { gas exchange, } \\
\text { and perceived } \\
\text { dyspnea (RPB) } \\
\text { during high } \\
\text { intensity exercise } \\
\text { between a } \\
\text { mouthpiece and a } \\
\text { face mask. }\end{array}$ & $\begin{array}{l}14 \text { trained men }(7 \\
\text { with a mask and } 7 \\
\text { with a mouthpiece) } \\
\text { with normal lung } \\
\text { function and who } \\
\text { reached a VO2 peak } \\
\text { of } 45 \mathrm{ml} \cdot \mathrm{kg}-1 \text {. } \\
\text { min }-1 \text { or higher. }\end{array}$ & $\begin{array}{l}\text { Randomized } \\
\text { group trial with a } \\
\text { mouthpiece (Hans } \\
\text { Rudolph) and a face } \\
\text { mask (7450, Hans } \\
\text { Rudolph) of } 6 \text { 'at } 90 \% \\
\text { V02 peak on a cycle } \\
\text { ergometer (Velotron } \\
\text { Pro, Racer Mate, Inc.). }\end{array}$ & $\begin{array}{l}\text { - Breathing mechanics } \\
\text { - Gas exchange variables } \\
\text { - Ratings of perceived } \\
\text { breathlessness (RPB) }\end{array}$ & $\begin{array}{l}\text { There were no significant differences } \\
\text { in respiratory mechanics, gas } \\
\text { exchange variables, or RPB. These } \\
\text { data suggest that the face mask can } \\
\text { be used interchangeably with the } \\
\text { mouthpiece and may even be a more } \\
\text { comfortable alternative during high } \\
\text { intensity exercise. }\end{array}$ \\
\hline
\end{tabular}


Table 2. Synthesis of results of the integrative review (continuation).

\begin{tabular}{|c|c|c|c|c|c|c|}
\hline Study & Methodological Rigor & Objective & Participants & Process & Measurement parameters & Results and conclusions \\
\hline $\begin{array}{l}\text { 8- Pifarré, F., Zabala, } \\
\text { D.D., Grazioli, G., \& } \\
\text { Maura, I. D. (2020). } \\
\text { Covid-19 and mask } \\
\text { in sports. }\end{array}$ & $\begin{array}{l}\text { - PEDro Scale: } 5 \\
\text { - oRecomendation: B } \\
\text { - Level of evidence:II-1 } \\
\text { - Internal validity: B } \\
\text { - External validity: no } \\
\text { - Quotes in scholar: } 3 \\
\text { - Magazine: Apunts } \\
\text { Medicina de l'Esport } \\
\text { - H index: } 10 \text { (Q4) }\end{array}$ & $\begin{array}{l}\text { To evaluate the } \\
\text { physiological } \\
\text { impact of } \\
\text { hypercapnia } \\
\text { and hypoxia } \\
\text { generated by } \\
\text { masks during } \\
\text { the practice of } \\
\text { aerobic sports. }\end{array}$ & $\begin{array}{l}8 \text { subjects ( } 6 \text { men } \\
\text { and } 2 \text { women) }\end{array}$ & $\begin{array}{l}\text { Ruffier protocol } \\
\text { through } 21 \text { push-ups } \\
\text { between } 6-8 \text { METS, } \\
\text { with and without a } \\
\text { mask, in the open } \\
\text { air. A pulse oximeter } \\
\text { (FS20C TEMPI-TEC) } \\
\text { and a gas analyzer } \\
\text { (MultiRae from Rae } \\
\text { Systems } ® \text { ) were } \\
\text { used. }\end{array}$ & $\begin{array}{l}\text { - Heart Rate (HR) } \\
\text { - } 02 \text { and CO2 concentration inside } \\
\text { the mask. } \\
\text { - Arterial } 02 \text { Saturation (Sat02). }\end{array}$ & $\begin{array}{l}\text { There was a decrease in } 02 \text { before } \\
\text { exercise }(20.9 \%) \text {, during }(18.3 \%) \text { and } \\
\text { post-exercise }(17.8 \%)(p<.001) \text {, an } \\
\text { increase in } C 02 \text { in the three previous } \\
\text { conditions }(464.14162,17000 \mathrm{ppm}, \\
\text { respectively; } p<.001) \text { and a decrease } \\
\text { in baseline } 02 \text { saturation }(97.6 \pm \\
1.5 \%) \text { compared to post-exercise } \\
\text { arterial } 02 \text { saturation }(92.1 \pm 4, \\
12 \%)(p<.02) \text {. The use of masks in } \\
\text { athletes reduces } 02 \text { by } 3.7 \% \text {, causes } \\
\text { hypoxic and hypercapnic respiration } \\
\text { in an effort of } 6-8 \text { METS. }\end{array}$ \\
\hline $\begin{array}{l}\text { 9- Tobin, B., } \\
\text { Costalat, G., \& } \\
\text { Renshaw, G.M.C. } \\
\text { (2020). Intermittent } \\
\text { not continuous } \\
\text { hypoxia provoked } \\
\text { haematological } \\
\text { adaptations in } \\
\text { healthy seniors: } \\
\text { hypoxic pattern } \\
\text { may hold the key }\end{array}$ & $\begin{array}{l}\text { - PEDro Scale: } 5 \\
\text { - }{ }^{\circ} \text { Recomendation: B } \\
\text { - Level of evidence: II-3 } \\
\text { - Internal validity: M } \\
\text { - External validity: no } \\
\text { - Quotes in scholar: } 1 \\
\text { - Magazine: European } \\
\text { Journal of Applied } \\
\text { Physiology } \\
\text { - H index: } 128 \text { (Q1) }\end{array}$ & $\begin{array}{l}\text { To investigate } \\
\text { the effect of two } \\
\text { hypoxic patterns } \\
\text { (continuous and } \\
\text { intermittent) on } \\
\text { hematological } \\
\text { adaptation, stress, } \\
\text { and heart damage } \\
\text { in healthy older } \\
\text { participants }\end{array}$ & $\begin{array}{l}15 \text { older } \\
\text { participants with no } \\
\text { previous disease }\end{array}$ & $\begin{array}{l}\text { Three-phase protocol } \\
\text { for } 3 \text { consecutive } \\
\text { weeks: } 5 \text { days outdoors } \\
\text { without a mask, } 5 \\
\text { days of respiration } \\
\text { with a normoxic mask } \\
(\text { FiO2 }=21 \%) \text {, and } 5 \\
\text { days of intermittent } \\
\text { hypoxia (HI) adapted } \\
\text { to achieve a medium } \\
\text { peripheral oxygen } \\
\text { saturation ( } \mathrm{SpO2)} \text { of } \\
85 \% \text { during } ~ 70 \text { 'of } \\
\text { cumulative exposure } \\
\text { to hypoxia. After a } \\
5 \text {-month washout } \\
\text { period, participants } \\
\text { were reminded to } \\
\text { perform continuous } \\
\text { hypoxia (CH, Sp02 = } \\
85 \%, \sim 70 \text { ). }\end{array}$ & $\begin{array}{l}\text { - Red blood cell count (RBCc), } \\
\text { - Hemoglobin concentration ([Hb]) } \\
\text { - Hematocrit (Hct) } \\
\text { - Percentage of reticulocytes (\% } \\
\text { Retics). } \\
\text { - Secretory immunoglobulin A } \\
\text { (S-IgA) } \\
\text { - Cortisol } \\
\text { - Cardiac troponin T (cTnT) }\end{array}$ & $\begin{array}{l}\text { RBCc only increased on day } 5 \text { of } \\
\text { Intermittent Hypoxia (IH) treatment } \\
\text { compared to baseline values on day } \\
5(+7.7 \%, \mathrm{p}<.01) \text { and sham values } \\
\text { on day } 5(+12.9 \%, \mathrm{p}<.01) \text {. Hb only } \\
\text { increased on day } 5 \text { of } \mathrm{HI} \text { treatment } \\
\text { compared to baseline values on day } \\
5(+14.7 \%, \mathrm{p}<0.01) \text { and sham } \\
\text { values on day } 5(+14.3 \%, \mathrm{p}<, 01) \text {. } \\
\text { No differences were observed in } \\
\mathrm{S}-\mathrm{IgA} \text {, cortisol, or } \mathrm{CTnT} \text { after HI or } \\
\text { Continuous Hypoxia }(\mathrm{CH}) \text {. These } \\
\text { results revealed that the inherent } \\
\text { differences in the hypoxic patterns } \\
\text { of HI and CH could provide crucial } \\
\text { components necessary to trigger } \\
\text { hematological changes in older } \\
\text { people, without eliciting immune } \\
\text { stress responses or damaging the } \\
\text { myocardium. }\end{array}$ \\
\hline $\begin{array}{l}\text { 10- Wong, A.Y. et } \\
\text { al. (2020). Impact } \\
\text { of the Covid-19 } \\
\text { pandemic on sports } \\
\text { and exercise }\end{array}$ & $\begin{array}{l}\text { - PEDro Scale: } 5 \\
\text { - 'Recomendation: A } \\
\text { - Level of evidence: I } \\
\text { - Internal validity: B } \\
\text { - External validity: si } \\
\text { - Quotes in scholar: } 0 \\
\text { - Magazine: Asia-Pacific } \\
\text { Journal of Sports } \\
\text { Medicine, Arthroscopy, } \\
\text { Rehabilitation and } \\
\text { Technology } \\
\text { - H index: } 8 \text { (Q3) }\end{array}$ & $\begin{array}{l}\text { Investigate the } \\
\text { physiological } \\
\text { effect of wearing } \\
\text { a mask during } \\
\text { exercise }\end{array}$ & $\begin{array}{l}23 \text { healthy } \\
\text { volunteer athletes } \\
(10 \text { men and } 13 \\
\text { women with a } \\
\text { mean age of } 33.8 \\
\text { years) }\end{array}$ & $\begin{array}{l}\text { Staggered treadmill } \\
\text { walks were } \\
\text { performed at } 4 \mathrm{~km} \\
/ \mathrm{h} \text { for } 6 \text { ' with and } \\
\text { without wearing a } \\
\text { surgical mask, and in } \\
\text { a random order with } \\
\text { sufficient rest time } \\
\text { between trials. }\end{array}$ & $\begin{array}{l}\text { - Heart Rate (HR) } \\
\text { - Rate of Perceived Effort (RPE) }\end{array}$ & $\begin{array}{l}\text { The heart rate of the subjects who } \\
\text { wore a mask was } 128 \text { beats per } \\
\text { minute and the PE was } 12.7 \text { out of } \\
20 \text {. In those without a mask, the } \\
\text { results were a heart rate of } 124 \\
\text { beats per minute and an RPE of } \\
\text { 10.8. Wearing a mask significantly } \\
\text { raises your heart rate and perceived } \\
\text { exertion. }\end{array}$ \\
\hline
\end{tabular}

oRecommendation: Good evidence for recommendation (A) / Moderate evidence (B) / Contradictory evidence (C) / Moderate evidence for no recommendation (D) / Good evidence for no recommendation (E) / Insufficient evidence for recommendation (I)

Level of evidence: with random assignment (I) / without random assignment (II-1) / existing evidence in control studies (II-2) / existing evidence with or without intervention without random assignment (II-3) / Existing evidence a based on the opinion of experts (III)

Internal validity: meets specific criteria of a well-designed study (B: Good) / does not meet at least one of the specific criteria, but without methodological defects (M: moderate) / study with at least one serious methodological defect (I: insufficient)

recommended that more established simulated altitude training methods be used when intermittent hypoxic training is incorporated. In this sense, the majority of studies in this area compare groups that use the ETM and controls to determine the effect on aerobic performance. In contrast, in the study of Bellovary et al. (2019) made comparisons with intermittent hypoxic training (IHT) revealing how ETM and IHT affect the economy of exercise. In the study of Bellovary et al. (2019) determined the effects on aerobic performance and economy in cyclists through intermittent hypoxic training (IHT) and ETM, the results showed that the IHT group, but not the ETM group, experienced an increase in glycolytic energy during submaximal exercise, that is, better lactic anaerobic performance. On the other hand, Tobin et al. (2020) studied the effect of two continuous $(\mathrm{CH})$ and intermittent $(\mathrm{HI})$ hypoxic patterns on hematological adaptation, stress, and cardiac damage in healthy older participants, concluding that the red blood cell count and hemoglobin concentration increased to the fifth day of HI treatment.

In the current pandemic situation, certain measures should be established for the use of different types of mask depending on the type and intensity of physical exercise to be performed. In the study of Guo and Fu (2019) they related the level of air pollution with the performance of the corridors through a mathematical model, they took into account the data of the daily air quality index and the meteorological conditions through Bloomberg, verifying that the concentrations on race days in different cities of China exceeded the standards established by the World Health Organization (WHO). Research is necessary along the lines of the articles analyzed in this review and the collection of information provided by organizations such as the WHO, NIOSH or the EU, to help stop the spread of both SARS-COV-2 during the practice of physical exercise. 


\section{Conclusions}

After reviewing the literature, it is highlighted that the use of masks in athletes causes hypoxic and hypercapnic respiration, being more evident in moderate to high intensity exercises of more than 6 METS. It could be said that wearing a mask like the Elevation Training Mask (ETM) does not appear to act as an altitude simulator, but rather as a respiratory muscle training device. In addition, Intermittent Hypoxic Training (IHT), in contrast to ETM, improves lactic anaerobic performance. There are no significant differences in gas exchange or breathing patterns using a mask or a nose clip and mouthpiece in stress tests, although, the use of a nose clip and mouthpiece in incremental tests could alter breathing patterns during exercise, and the mask, in addition to being more comfortable, allows you to reach a constantly higher peak of power. Finally, following the considerations of several authors (Toresdahl \& Asif, 2020; Wong et al., 2020) and also following the results of the studies analyzed in this integrative review, asymptomatic athletes should not be recommended to wear a mask while perform physical exercise to avoid infection with Covid-19 in the community setting or while traveling, as the risk of infection is not significantly reduced and would increase the physiological burden on the body, especially in those with multiple underlying comorbidities.

\section{Limitations and future lines of research}

The size of the samples of most of the studies analyzed is not representative and data on the characteristics of the different types of masks used are not provided, which entails a high degree of subjectivity and the conclusions may not be extrapolated to the state of the art. Neither results are shown on heterogeneity between the results of the control / experimental group of the different studies analyzed. Along these lines, the results of this review should be combined through a meta-analysis that allows assessing discrepancies in clinical (participants, interventions, outcomes), methodological (design, performance, analysis strategy) and statistical (random variability) heterogeneity sample). Moreover the methodological quality of the studies included in the analysis of the results has not been agreed between two or more reviewers. Although the studies analyzed in the review meet external validity criteria, have a high level of scientific evidence and a good degree of recommendation, higher-level references indexed in JCR are missing in this field of action.

On the one hand, research is needed in instruments that help to monitor the flow and volume of respiratory air, in line with the study by Zhou, Costa and Lukowicz (2020) in which they conclude that barometers with millimeter fingerprints embedded in masks can function in a similar to a digital spirometer to aid in monitoring. Playing sports with a mask is possible, but breathing could deteriorate during prolonged physical exercise, a protocolized investigation would be useful to avoid any risk of contagion, which studies the implication of the use of different types of masks in oxygen saturation, acidification in the blood and respiratory efficiency. On the other hand, future investigations are being planned that use different types of masks at different heights as a method of training respiratory muscles, and that are validated through stress tests and direct gas measurement, in this way it will be possible to know more exactly an improvement over the VO2max value due to the greater inspiratory and expiratory effort. Finally, it should be noted that there are very few comparative studies on respiratory protection devices during physical exercise, it would be interesting to know the different conditions of hypoxia and hypercapnia analyzed through the graduation of filters or the particularities of each mask while performing physical exercise for a long time. 


\section{REFERENCES}

Bellovary, B.N., King, K.E., Nunez, T.P., Mccormick, J.J., Wells, A.D. Bourbeau, K.C., Fennel, Z.J., Li, Z., Johnson, K.E., Moriarty, T., \& Mermier, C.M. (2019). Effects of high-intensity interval training while using a breathing-restrictive mask compared to intermittent hypobaric hypoxia. Journal of Human Sport and Exercise, 14(4), 821-833. doi:10.14198/jhse.2019.144.11

Birtwhistle, R., Pottie, K., Shaw, E., Dickinson, J.A., Brauer, P., Fortin M., Bell, N., Singh, H., Tonelli, M., Connor,S., Lewin, G., Joffres, M. \& Parkin, P. (2012). Canadian Task Force on Preventive Health Care: we're back! Canadian Family Physician, 58, 13-5.

Bourouiba, L. (2020). Turbulent Gas Clouds and Respiratory Pathogen Emissions Potential Implications for Reducing Transmission of Covid-19. JAMA The Journal of the American Medical Association, 323(18), 1837-1838. doi:10.1001/jama.2020.4756

Chen, Y., Ebenstein, A.M., \& Greenstone, H.L. (2013). Evidence on the impact of sustained exposure to air pollution on life expectancy from China's Huai River policy, Proceedings of the National Academy of Sciences of the United States of America 110, 12936-12941.

Ciapponi, A. (2015). QUADAS-2: instrumento para la evaluación de la calidad de estudios de precisión diagnóstica. Revista Evidencia Actualización en la Práctica Ambulatoria, 18(1), 22-26.

Del Río Haza, F., López, O.G., Tobón, S.H., Leyva, P.D., \& García, R.S. (2020). Los aerosoles y los virus. II. La física del contagio aéreo de la Covid-19. Departamento de Física, Universidad Autónoma Metropolitana, Iztapalapa.

Freemas, J.A., Wilhite, D.P, Greenshields, J.T., Adamic, E.M., \& Mickleborough, T.D. (2020) Comparison between a facemask and mouthpiece on breathing mechanics and gas exchange variables during high-intensity exercise. European Journal of Sport Science, 20(2), 211218, doi:10.1080/17461391.2019.1628309

Guo, M., \& Fu, S. (2019). Running With a Mask? The Effect of Air Pollution on Marathon Runners' Performance. Journal of Sports Economics, 20(7), 903-928. doi:10.1177/1527002518822701

Hutton, B., Salanti, G., Caldwell, D. M., Chaimani, A., Schmid, C. H., Cameron, C., Loannidis, J. P. A., Straus, S., Thorlund, K., Jansen, J. P., Mulrow, C., Catalá-López, F., Gøtzsche, P. C., Dickersin, K., Boutron, I., Altman, D. A., \& Moher, D. (2015). The PRISMA extension statement for reporting of systematic reviews incorporating network metaanalyses of health care interventions: Checklist and explanations PRISMA extension for network meta-analysis. Annals of Internal Medicine, 162(11), 777-784. doi:10.7326/M14-2385

Jung, H.C., Lee, N.H., John, S.D., \& Lee, S. (2019). The elevation training mask induces modest hypoxaemia but does not affect heart rate variability during cycling in healthy adults. Biology of Sport, 36(2), 105-112. doi:10.5114/biolsport.2019.79976

Kao, T. W., Huang, K. C., Huang, Y. L., Tsai, T. J., Hsieh, B. S., \& Wu, M. S. (2004). The physiological impact of wearing an N95 mask during hemodialysis as a precaution against SARS in patients with end-stage renal disease. Journal of the Formosan Medical Association, 103(8), 624-628.

Leung, N., Chu, D., Shiu, E., Chan, K. H., McDevitt, J. J., Hau, B., Yen, H. L., Li, Y., Ip, D., Peiris, J., Seto, W. H., Leung, G. M., Milton, D. K., \& Cowling, B. J. (2020). Respiratory virus shedding in exhaled breath and efficacy of face masks. Nature medicine, 26(5), 676-680. doi:10.1038/ s41591-020-0843-2
National Institute of Occupational Safety and Health (NIOSH). (2020). Approval Support, Test Procedures, Standards, and User Notices. Department of Health \& Human Services, U.S.A. Recuperado de https://www.cdc.gov/niosh/npptl/respmanuf.html

Organización Mundial de la Salud. (2020). Advice on the use of masks in the context of Covid-19: interim guidance, 5 June 2020. World Health Organization. Recuperado de https://apps.who.int/iris/ handle/10665/332293

Pifarré, F., Zabala, D.D., Grazioli, G., \& Maura, I. D. (2020). Covid-19 and mask in sports. Apunts Sports Medicine. doi:10.1016/j. apunsm.2020.06.002

Porcari, J.P., Probst, L., Forrester, K., Doberstein, S., Foster, C., Cress, M.L., \& Schmidt, K. (2016). Effect of wearing the elevation training mask on aerobic capacity, lung function, and hematological variables. Journal of Sports Science and Medicine, 15(2), 379-386.

Pun, V. C., J., \& Manjouride, H.S. (2017). Association of ambient air pollution with depressive and anxiety symptoms in older adults: results from the NSHAP study. Environmental Health Perspectives 125, 342 348.

Rundell, K. W. (2012). Effect of air pollution on athlete health and performance. British Journal of Sports Medicine 46, 407-412.

Sellers, J.H., Monaghan, T.P., Schnaiter, J.A., Jacobson, B.H., \& Pope, Z.K. (2016). Efficacy of a Ventilatory Training Mask to Improve Anaerobic and Aerobic Capacity in Reserve Officers' Training Corps Cadets. Journal of Strength and Conditioning Research, 30(4), 1155-1160. doi:10.1519/JSC.0000000000001184

Tobin, B., Costalat, G., \& Renshaw, G.M.C. (2020). Intermittent not continuous hypoxia provoked haematological adaptations in healthy seniors: hypoxic pattern may hold the key. European Journal of Applied Physiology, 120, 707-718. doi:10.1007/s00421-020-04310-y

Toresdahl B.G., \& Asif, I.M. (2020). Coronavirus Disease 2019 (Covid-19) Considerations for the Competitive Athlete. Sports Health, 12(3), 221-224. doi:10.1177 / 1941738120918876

Wagner, D.R., \& Clark, N.W. (2016). Similar results for face mask versus mouthpiece during incremental exercise to exhaustion. Journal of Sports Sciences, 34(9), 852-855

Whittemore, R, Chao, A, Jang, M., Minges, K.E., \& Park, C. (2014). Methods for knowledge synthesis: an overview. Heart Lung,43(5), 453-61. doi:10.1016/j.hrtlng.2014.05.014

Wong, A. Y., Ling, S. K., Louie, L. H., Law, G. Y., So, R.C., Lee, D.C., Yau, F.C., \& Yung, P.S. (2020). Impact of the Covid-19 pandemic on sports and exercise. Asia-Pacific Journal of Sports Medicine, Arthroscopy, Rehabilitation and Technology, 22, 39-44. doi:10.1016/j. asmart.2020.07.006

Zhou, B., Costa, A. B., \& Lukowicz, P. (2020). Accurate spirometry with integrated barometric sensors in face-worn garments. Sensors (Switzerland), 20(15), 1-22. doi:10.3390/s20154234

Zhu, N., Zhang, D., Wang, W., Li, X., Yang, B., Song, J., Zhao, X., Huang, B., Shi, W., Lu, R., Niu, P., Zhan, F., Ma, X., Wang, D., Xu, W., Wu, G., Gao, G. F., Tan, W., \& China Novel Coronavirus Investigating and Research Team (2020). A Novel Coronavirus from Patients with Pneumonia in China, 2019. The New England journal of medicine, 382(8), 727-733. doi:10.1056/NEJMoa2001017 